\title{
具有表皮生长因子受体抑制活性的喹唑啉衍生物研究进展
}

\author{
张 英王培义胡德禹金林红杨 松*
}

(贵州大学精细化工研究中心 绿色农药与农业生物工程国家重点实验室培育基地与教育部重点实验室

贵阳 550025)

\begin{abstract}
摘要 综述了近十年来具有表皮生长因子受体(EGFR)抑制活性的喹唑啉衍生物的国内外研究进展, 按照喹唑啉环上不 同位置上的取代基类型分为四类, 介绍了化合物不同位置上取代基对此类化合物生物活性的影响, 并对其发展趋势和 应用前景作了展望.

关键词 表皮生长因子受体; 喹唑啉; 生物活性
\end{abstract}

\section{Research Progress on Quinazoline Derivatives with Epidermal Growth Factor Receptor Inhibiting Bioactivity}

\author{
Zhang, Ying Wang, Peiyi Hu, Deyu Jin, Linhong Yang, Song* \\ (State Key Laboratory Breeding Base of Green Pesticide and Agricultural Bioengineering, Center for Research and Develop- \\ ment of Fine Chemicals, Guizhou University, Guiyang 550025)
}

\begin{abstract}
The progress on quinazoline derivatives with epidermal growth factor receptor (EGFR) inhibiting bioactivity is reviewed in this paper. Quinazoline compounds are classified to four types according to different substituent groups at different ring position. The effects of substituent groups at different ring positions on the bioactivities are discussed. And the development tendency as well as the prospect of quinazoline derivatives as epidermal growth factor receptor tyrosine kinase inhibitors is summarized.
\end{abstract}

Keywords epidermal growth factor receptor; quinazoline; bioactivity

表皮生长因子受体(EGFR)是一种受体酪氨酸激酶, 能激活并诱导癌细胞增殖、存活和迁移的信号路径, 从 而导致各种表皮癌(如皮肤癌、乳腺癌、卵巢癌以及和 非小细胞肺癌、头颈部肿瘤、肠癌、膀胱癌)的发生 ${ }^{[1 \sim 5]}$. EGFR 是一种催化性的跨膜受体蛋白，在细胞膜内侧有 一个催化区域, 细胞膜外侧有一个配体结合区域. 当配 体也就是表皮生长因子结合到结合区域时 EGFR 被激 活，而与配体竞争结合位点或直接干扰 EGFR 的催化位 点可抑制 EGFR 的活性 ${ }^{[6]}$. 自从 1994 年 Fry 等 ${ }^{[7]}$ 发现 4苯胺基喹唑啉(PD 153035)作为 EGFR 酪氨酸激酶的特 异性抑制剂以来, 喹唑啉类化合物成为抗癌药物研制的 热点之一 ${ }^{[8 \sim 11]}$, 已发展出第一代及第二代的靶标药物. 第一代EGFR 可逆抑制剂吉非替尼 ZD1839 (Iressa) ${ }^{[12 ~ 16]}$

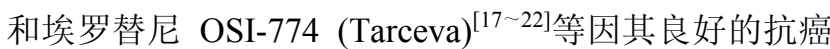
活性已经商品化. 然而, 它们对癌细胞生长仅产生抑制 效果, 无法有效地灭杀癌细胞, 且长期使用会导致肿瘤 的耐药 ${ }^{[23]}$, 因此它们在临床上的效果受到限制, 这就促 使了第二代 EGFR 不可逆抑制剂的研发. 目前, 第二代 对 EGFR 有不可逆抑制效果的药物也已进入临床测试阶 段, 如 Boehringer Ingelheim 开发的 BIBW2992 已经进 入临床 II 期 ${ }^{[24]}$, 且可与其他治疗方法相结合进行组合治 疗, 效果更佳 ${ }^{[25]}$. 本文综述了近十年来国内外研究发现 的有 EGFR 抑制活性的喹唑啉衍生物, 并按其结构进行 分类，希望能找到结构和活性之间的关系，发现抗肿瘤 活性更高、更安全的喹唑啉类 EGFR 抑制剂.

\footnotetext{
*E-mail: jhzx.msm@gmail.com

Received August 6, 2011; revised October 10, 2011; accepted November 4, 2011.
}

Project supported by the National Key Science Project for Basic Research (Nos. 2010CB126105, 2010CB134504), the National Natural Science Foundation of China (No. 21172048), and the National Key Technology Research \& Development Program (Nos. 2011BAE06B02, 2011BAE06B05).

国家重点基础研究发展计划(973) (Nos. 2010CB126105, 2010CB134504)、国家自然科学基金(No. 21172048)和国家科技支撑计划课题(Nos. 2011BAE06B02, 2011BAE06B05)资助项目. 
<smiles>COc1cc2ncnc(Nc3cccc(Br)c3)c2cc1OC</smiles>

PD153035<smiles>C#Cc1cccc(Nc2ncnc3cc(OCCOC)c(OCCOC)cc23)c1</smiles>

ZD1839 (Iressa, Gefitinib)<smiles>COc1cc2ncnc(Nc3ccc(F)c(Cl)c3)c2cc1OCCCN1CCOCC1</smiles>

OSI-774 (Tarceva, Erlotinib)<smiles>CN(C)C/C=C/C(=O)Nc1cc2c(Nc3ccc(F)c(Cl)c3)ncnc2cc1OC1CCOC1</smiles>

BIBW2992

\section{6-取代-7-烷氧基-4-芳胺基喹唑啉}

\section{$1.16,7$-二烷氧基-4-芳胺基喹唑啉}

2003 年 Vicentini 等 ${ }^{[26]}$ 研究了 EGFR 酪氨酸激酶抑 制剂 ZD1839 (Gefitinib)对体外前列腺癌细胞(包括非雄 激素依赖型的 PC3, DU145, TSU-Pr1 癌细胞和雄激素依 赖型的 ND1, LNCaP, LALVA-31 癌细胞)增殖活性的影 响, 发现 ZD1839 抑制上述肿瘤细胞的 EGFR 自磷酸化 的 $\mathrm{IC}_{50}$ 值范围为 $0.46 \sim 0.97 \mu \mathrm{mol} / \mathrm{L}$, 对细胞增殖抑制的 $\mathrm{IC}_{50}$ 值范围为 $0.37 \sim 1.03 \mu \mathrm{mol} / \mathrm{L}$. 在剂量大于 $1 \mu \mathrm{mol} / \mathrm{L}$ 时, ZD1839 可诱导 PC3 细胞的调亡. 研究结果表明此类 EGFR 酪氨酸激酶抑制剂可能在早期就能阻断人前列腺 癌细胞的生长及增殖.

2004 年 Shreder 等 ${ }^{[27]}$ 将荧光物质四甲基罗丹明 (TAMRA)引入含叠氮基团的喹唑啉结构中, 合成了一 种作用于 EGFR 的光亲和性苂光探针 AX7593, 二者结 合常数为 $280 \mathrm{nmol} / \mathrm{L}$, 对 EGFR 标记的光亲和性的有效 率达到 34\%, 其有效性与 EGFR 抑制剂 PP2 相当, 可用 于建立非放射性、高通量笁选方法以发现新的 EGFR 抑
制剂.<smiles>COc1cc2c(Nc3cccc(N)c3)ncnc2cc1OCCOCCOCCOCCNC(C)(C)C</smiles>

2005 年 Hennequin 和 Halsall ${ }^{[28]}$ 在专利中报道了一系 列喹唑啉化合物 1, 与 OSI-774 相比, 主要是固定喹唑 啉环 6-位为甲氧基取代，而 7-位则改造为含有酰胺基吗 啉结构的烷氧基取代，该系列化合物对人口腔上皮癌 $(\mathrm{KB})$ 细胞 $\mathrm{EGFR}$ 具有较好的抑制活性, $\mathrm{IC}_{50}$ 值范围为 $0.001 \sim 5 \mu \mathrm{mol} / \mathrm{L}$, 活体试验表明适合的口服剂量为 $1 \sim$ $200 \mathrm{mg} \cdot \mathrm{kg}^{-1} \cdot \mathrm{d}^{-1}$. 其中化合物 $\mathbf{1 a} \sim \mathbf{1 e}$ 抑制 $\mathrm{EGFR}$ 的 $\mathrm{IC}_{50}$ 值分别为 $112,55,37,84,109 \mathrm{nmol} / \mathrm{L}$, 抑制酪氨酸激酶 磷酸化的 $\mathrm{IC}_{50}$ 值分别为 $76,41,30,65,52 \mathrm{nmol} / \mathrm{L}$, 具有 进一步开发成癌症治疗药物的潜力.<smiles>[R]c1cc2ncnc(Nc3cccc(Cl)c3F)c2cc1OC</smiles><smiles>[R]OCOCC(=O)N1CCC(OC(C)(C)C)CC1</smiles>

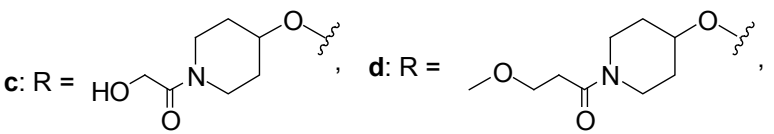<smiles>[R]=COC1CCN(C(=O)CCOC(C)C)CC1</smiles>

2005 年 Bradbury 等 ${ }^{[29]}$ 在专利中报道了一系列喹唑 啉化合物 2, 该系列化合物喹唑啉环 7-位仍为甲氧基, 而 6-位则是含有酰胺基的哌啶氧基取代，对 $\mathrm{KB}$ 细胞 EGFR 的 $\mathrm{IC}_{50}$ 值范围为 $0.001 \sim 10 \mu \mathrm{mol} / \mathrm{L}$, 活体试验表 明适合的口服剂量为 $1 \sim 200 \mathrm{mg} \bullet \mathrm{kg}^{-1} \cdot \mathrm{d}^{-1}$. 其中化合物 2a 2c 抑制酪氨酸激酶蛋白质磷酸化的 $\mathrm{IC}_{50}$ 值分别为 4, 3, $4 \mathrm{nmol} / \mathrm{L}, \mathbf{2 a}$ 和 $\mathbf{2 b}$ 抑制 $\mathrm{EGFR}$ 的 $\mathrm{IC}_{50}$ 值分别为 9,17 $\mathrm{nmol} / \mathrm{L}$ ，具有进一步开发成癌症治疗药物的潜力. 
<smiles>CNC(=O)CN1CCC(Oc2cc3c(Nc4ccc(F)c(Cl)c4F)ncnc3cc2OC)CC1</smiles><smiles>COc1cc2ncnc(Nc3cccc(Cl)c3F)c2cc1OC1CCN(CC(=O)NCCN2CCCC2)CC1</smiles>

2b<smiles>COc1cc2ncnc(Nc3cccc(Cl)c3F)c2cc1OC1CCN(CC(=O)N2CCN(C)CC2)CC1</smiles>

2c

2005 年 Barlaam 等 ${ }^{[30]}$ 在专利中报道了一系列喹唑 啉化合物 3 , 与前述化合物 2 相比, 其结构特征主要是 在 6,7-位的酰胺基哌啶氧基中引入噻唑、异啞唑、吡啶 等杂环基团，其中化合物 $\mathbf{3 a} \sim \mathbf{3 f}$ 抑制 $\mathrm{EGFR}$ 的 $\mathrm{IC}_{50}$ 值分 别为 $17,16,47,87,30,17 \mathrm{nmol} / \mathrm{L}$, 对酪氨酸激酶蛋白质 磷酸化的 $\mathrm{IC}_{50}$ 值分别为 $<1,14,15,43,<1,<1 \mathrm{nmol} / \mathrm{L}$, 具备良好的开发潜力.<smiles>[R]c1cc2ncnc(Nc3cccc(Cl)c3F)c2cc1[R]</smiles><smiles>[R1]O[CH]OC[C@@H]1ON=C(C)C1NCC(=O)N1CCC(OC)CC1</smiles><smiles>COC1CCN(C(=O)c2ccno2)CC1</smiles><smiles>[R]OCOCCOC</smiles>

胆囊癌(BTC)是一种存活率非常低的疾病, 对胆囊 癌并无有效的治疗手段或药物. 2005 年 Kiguchi 等 ${ }^{[31]}$ 报 道了 Gefitinib 作为口服性 EGFR 酪氨酸激酶抑制剂对转 人胆囊癌(BTC)基因小鼠的化学预防和治疗效果, 结果 表明，每天在小鼠的饮食中加入 $400 \mathrm{mg} \bullet \mathrm{kg}^{-1} \mathrm{Gefitinib,}$ 对转人胆道癌(BTC)的小鼠具有良好的的化学预防和治 疗作用, 研究同时也证实 EGFR 的激活对 BTC 的发展中
起着重要作用, 而以 EGFR 为靶标可能是治疗胆道癌的 有效途径.

2005 年 Hennequin 和 Halsall ${ }^{[32]}$ 报道了一系列喹唑啉 化合物 $\mathbf{4}$, 与化合物 3 相比, 该类化合物的特征主要是 在 7-烷氧基中, 将六元哌啶环改造为五元或四元氮杂 环，该系列化合物抑制 $\mathrm{KB}$ 细胞 $\mathrm{EGFR}$ 的 $\mathrm{IC}_{50}$ 值范围为 $0.001 \sim 5 \mu \mathrm{mol} / \mathrm{L}$, 活体试验表明适合的口服剂量为 $1 \sim$ $200 \mathrm{mg} \cdot \mathrm{kg}^{-1} \cdot \mathrm{d}^{-1}$. 其中代表性的化合物 $\mathbf{4 a} \sim \mathbf{4 d}$ 抑制 EGFR 的 $\mathrm{IC}_{50}$ 值分别为 $71,48,119,397 \mathrm{nmol} / \mathrm{L}$ ，抑制酪 氨酸激酶蛋白质磷酸化的 $\mathrm{IC}_{50}$ 值分别为 $17,8,217,41$ $\mathrm{nmol} / \mathrm{L}$, 该系列化合物具有进一步开发成癌症治疗药物 的可能<smiles>[R]c1cc2ncnc(Nc3cccc(Cl)c3F)c2cc1OC</smiles>

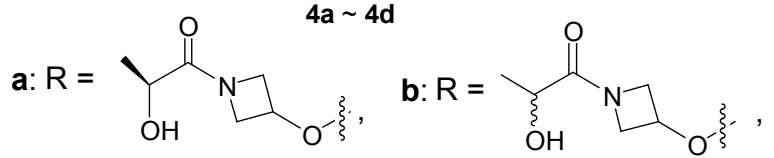

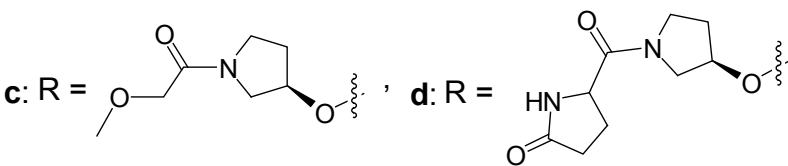

2006 年 Halsall 小组 ${ }^{[33]}$ 在化合物 4 基础上进一步进 行结构改造, 在喹唑啉环 C-6 位连接的氧原子上引入 1甲基-2-甲酰胺哌啶取代基才，设计合成了一系列喹唑 啉化合物 5, 测试了该系列化合物对 human nasopharangeal carcinoma (KB)细胞 EGFR 的活性, 其中化合物 5a 5c 对 EGFR 抑制活性的 $\mathrm{IC}_{50}$ 值分别为 56, 61, 23 $\mathrm{nmol} / \mathrm{L}$.<smiles>COc1cc2ncnc(Nc3cccc(Cl)c3F)c2cc1OC1CCN(C)[C@H](C(N)=O)C1</smiles><smiles>CNC(=O)[C@@H]1CC(Oc2cc3c(Nc4cccc(Cl)c4F)ncnc3cc2OC)CN(C)C1</smiles>

$5 b$<smiles>CNC(=O)[C@@H]1C[C@@H](Oc2cc3c(Nc4cccc(Cl)c4F)ncnc3cc2OC)CN(C)C1</smiles>

$5 c$ 
2007 年许传莲等 ${ }^{[34]}$ 合成了一系列 6,7-二甲氧基-4芳胺基喹唑啉衍生物 6, 对 EGFR-TK 有明显的抑制活 性. 研究发现 4-位氮上甲基取代对其活性影响不大, 而 4-位芳环上有 $\mathrm{Br}, \mathrm{Cl}$ 取代基或无取代基时，化合物对 EGFR 抑制作用较强; 4-位芳环上有甲氧基时，化合物对 EGFR 活性抑制作用较弱. 在 200 和 $40 \mu \mathrm{mol} / \mathrm{L}$ 浓度下, 化合物 $\mathbf{6 a} \sim \mathbf{6} \mathbf{c}$ 对 EGFR 酪氨酸激酶的抑制活性( $O D$ 值) 分别为 $0.1872,0.1921,0.1984$ 和 $0.2032,0.2448,0.2368$, 而 Gefitinib 的 OD 值为 $0.2416,0.2976$, 其抑制活性高于 对照药物 Gefitinib, 活性数据证明 4-位苯环上的取代基 变化对活性具有显著影响.<smiles>COc1cc2ncnc(N(C)c3ccccc3)c2cc1OC</smiles>

$6 a$<smiles>COc1cc2ncnc(Nc3ccccc3)c2cc1OC</smiles>

$6 b$<smiles>COc1cc2ncnc(Nc3ccccc3Cl)c2cc1OC</smiles>

2008 年 Breza 等 ${ }^{[35]}$ 设计合成了一系列喹唑啉类衍生 物 7, 并测试了其对 EGFR 野生型(WT-EGFR)和 EGFR 突变型 delL747-P753insS (DL-EGFR), L858R (LREGFR), L858R/T790M (LR/TM-EGFR)的抑制活性, 测 试结果表明, 该系列中化合物 7a $\sim 7 \mathbf{c}$ 对 EGFR 有较好的 抑制活性. 在 10 和 $30 \mu \mathrm{mol} / \mathrm{L}$ 浓度下, 对照药物 Gefitinib 和 Erlotinib 对 WT-EGFR, DL-EGFR 和 LREGFR 的抑制率范围分别为 $91 \% \sim 100 \%$ 和 $78 \% \sim 100 \%$, 但这两种对照药物对突变型 LR/TM- EGFR 没有抑制活 性; 化合物 7a 和 7b 对 WT-EGFR, DL-EGFR 和 LREGFR 三种 EGFR 有一定的抑制活性, 抑制率范围分别 为 $38 \% \sim 100 \%$ 和 $41 \% \sim 100 \%$, 而 $7 \mathbf{b}$ 在 $30 \mu \mathrm{mol} / \mathrm{L}$ 浓度 下还对 LR/TM-EGFR 有一定抑制活性，达 $22 \%$. 化合物 $7 \mathbf{c}$ 的抑制活性较好, 在同样浓度下抑制三种 EGFR 的活 性和 Erlotinib 相当, 比 Gefitinib 略差, 在 10 和 $30 \mu \mathrm{mol} / \mathrm{L}$ 浓度下对三种 EGFR 抑制率范围分别为 $60 \%$ 97\%和 $90 \% \sim 100 \%$, 此外, $7 \mathbf{c}$ 对突变型的 LR/TM-EGFR 具有良 好的抑制活性, 在 $30 \mu \mathrm{mol} / \mathrm{L}$ 浓度下对 LR/TM-EGFR 的
抑制率达到 $64 \%$, 比两种对照药物更具有优势.<smiles>Oc1ccc(Cl)cc1Nc1ncnc2ccccc12</smiles>

$7 a$<smiles>COc1cc2ncnc(NCC3CCCCC3)c2cc1OC</smiles>

7b<smiles>COc1cc2ncnc(Nc3ccc4c(c3)OCO4)c2cc1OC</smiles>

2008 年 Lee 等 ${ }^{[36]}$ 合成了 140 余个喹唑啉化合物 8, 生物活性测试表明该类化合物能有效抑制 EGFR 和突变 的 EGFR 过度表达诱导的癌细胞. 该系列化合物均有良 好的抑制 A431 和 SK-Br3 细胞的活性, 其中 $\mathbf{8 a}, \mathbf{8 b}$ 的活 性最好, 其 $\mathrm{IC}_{50}$ 值均为 $0.3 \mu \mathrm{mol} / \mathrm{L}$, 明显优于对照药物 Gefitinib (对 $\mathrm{A} 431$ 和 $\mathrm{SK}-\mathrm{Br} 3$ 细胞生长的 $\mathrm{IC}_{50}$ 值分别为 28 和 $206 \mu \mathrm{mol} / \mathrm{L}$ ). 与此同时, 测试了化合物 8 抑制 EGFR 和突变型 EGFR-T790M 的活性, 结果表明对照药 物 Gefitinib 抑制 $\mathrm{EGFR}$ 和 $\mathrm{T} 790 \mathrm{M}$ 的 $\mathrm{IC}_{50}$ 值分别为 639 和 $>5000 \mu \mathrm{mol} / \mathrm{L}$, 而化合物 $8 \mathrm{a}$ 抑制 EGFR 和 T790M 的 $\mathrm{IC}_{50}$ 值分别为 0.7 和 $0.8 \mu \mathrm{mol} / \mathrm{L}$. 进一步测试 $8 \mathbf{a}, 8 \mathbf{b}$ 抑制 A431 细胞的 EGFR 磷酸化的活性, 与可逆抑制剂 Erlotinib 相比较, 发现化合物抑制 $50 \%$ 的 EGFR 磷酸化 的时间超过 $24 \mathrm{~h}$ 甚至更长, 表明其为不可逆抑制剂.<smiles>C=CC(=O)N1CCC(Oc2cc3c(Nc4ccc(F)c(Cl)c4F)ncnc3cc2OC)CC1Oc1cc2c(Nc3ccc(F)c(Cl)c3F)ncnc2cc1OC</smiles>

8b 
2008 年 Schettino 等 ${ }^{[37]}$ 总结了 Erlotinib 作为 EGFR 受体酪氨酸激酶抑制剂在非小细胞性肺癌治疗中的应 用. Erlotinib 在一阶段临床试验中用于治疗病人的晚期 癌症 ${ }^{[38,39]}$, 其疗效与 Gefitinib 相似, 口服剂量为 150 $\mathrm{mg} / \mathrm{day}$ 时有效率达 $46 \%$. 二阶段临床试验中单独使用 时其有效率为 $31.3 \%{ }^{[40]}$, 二阶段临床试验中还尝试将 Erlotinib 与贝伐单抗药物(Bevacizumab, 商品名 Avastin, 重组人类单克隆 $\mathrm{IgG} 1$ 抗体，具有抑制人类血管内皮生 长因子的活性)进行组合治疗, 有效率达 $54 \%{ }^{[41]}$. 三阶 段临床试验中开展了 Erlotinib 与紫杉醇、卡铂等组合治 疗试验，发现组合并未显现明显优势[42]

2008 年 Ban 等 ${ }^{[43]}$ 合成了一系列含不饱烯烃的 4-芳 胺基喹唑啉 9, 其中 9a $\sim 9 \mathrm{c}$ 具有选择性的抑制 EGFR 过 度表达的 $\mathrm{A} 431$ 细胞增殖活性, 其 $\mathrm{GI}_{50}$ 值分别为 $0.6,1.14$ 和 $0.23 \mu \mathrm{mol} / \mathrm{L}$. 根据免疫印迹分析, 9a $\sim 9 \mathrm{c}$ 在 $1 \mu \mathrm{mol} / \mathrm{L}$ 浓度下能抑制 EGF 诱导的磷酸化, 其抑制率分别为 $71.3 \%, 81.6 \%, 92.9 \%$.<smiles>C=Cc1cccc(Nc2ncnc3cc(OCCOC)c(OCCOC)cc23)c1</smiles><smiles>C=C=Cc1cccc(Nc2ncnc3cc(OC)c(OC)cc23)c1</smiles><smiles>C=C=Cc1cccc(Nc2ncnc3cc(OCCOC)c(OCCOC)cc23)c1</smiles>

2008 年 Lueth 和 Loewe ${ }^{[44]}$ 用交叉偶联法合成了两个 4-吲哚取代的喹唑啉化合物 10a 和 $10 \mathrm{~b}$, 生物活性测试 表明, $10 \mathrm{a}$ 和 $10 \mathrm{~b}$ 抑制 $\mathrm{EGFR}$ 酪氨酸激酶的 $\mathrm{IC}_{50}$ 值分别 为 333 和 $131 \mathrm{nmol} / \mathrm{L}$, 与对照药物 Gefitinib 和 Erlotinib 的 $\mathrm{IC}_{50}$ 值分别为 33 和 $2 \mathrm{nmol} / \mathrm{L}$ 相比, 这两个化合物虽 然在活性上没有优势, 但在紫外下显强荧光, 其苂光强 度与奎宁硫酸盐相当, 可进一步发展为 EGFR 的显像剂.<smiles>COc1cc2ncnc(-c3c[nH]c4cc(F)c(Cl)cc34)c2cc1OCCCN1CCOCC1</smiles>

10a<smiles>COc1cc2ncnc(-c3c[nH]c4ccc(Br)cc34)c2cc1OCCCN1CCOCC1</smiles>

2008 年 Qian 和 $\mathrm{Cai}^{[45]}$ 报道了 6-位烷氧基中烷基部 分末端羧基取代的 8 个喹唑啉类化合物 11, 生物活性测 试结果表明, 化合物 11a 11c 对 EGFR 抑制活性的 $\mathrm{IC}_{50}$ 值均小于或等于 $0.1 \mu \mathrm{mol} / \mathrm{L}$.

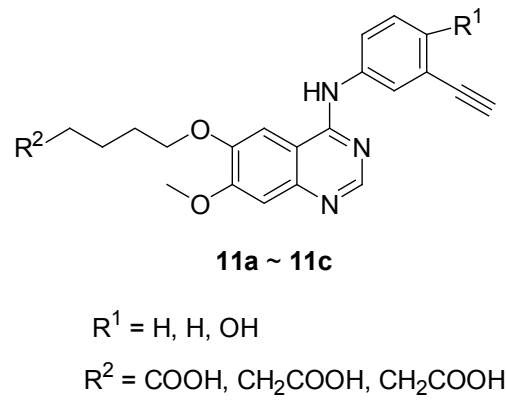

2008，2009 年 Cai 和 Qian 等 ${ }^{[46 ~ 48]}$ 在相关专利中报 道了一系列 6-位烷氧基中烷基部分末端为 $N$-手基基酰胺 的喹唑啉化合物，其中化合物 $12 \mathrm{a}$ 和 $\mathbf{1 2 b}$ 具有极好的抗 癌活性，对 EGFR 的抑制活性远优于 Erlotinib, 其 $\mathrm{IC}_{50}$ 值分别为 3.1 和 $2.4 \mathrm{nmol} / \mathrm{L}$, 而 Erlotinib 的 $\mathrm{IC}_{50}$ 值为 48 $\mathrm{nmol} / \mathrm{L}$; 此外, 化合物 $\mathbf{1 2 b}$ 抑制乳腺癌 (MCF-7, MDAMB468)、肺癌(H-292)和胰腺癌细胞(BXPC3) 的 $\mathrm{IC}_{50}$ 值均小于 $0.1 \mu \mathrm{mol} / \mathrm{L} ; \mathbf{1 2 b}$ 还具有诱导结肠癌细胞和 乳腺癌细胞凋亡的活性, Erlotinib 在浓度低于 $20 \mu \mathrm{mol} / \mathrm{L}$ 会失活, 而 $\mathbf{1 2 b}$ 在浓度低于 $0.17 \mu \mathrm{mol} / \mathrm{L}$ 才会失活. 令人 惊讶的是，研究还表明 12b 对 Erlotinib 耐药性的癌细胞 也有较好抑制活性. 该系列化合物变成酒石酸盐后溶解 性更好, 其中化合物 $\mathbf{1 2 b}$ 酒石酸盐的溶解度为 60 $\mathrm{mg} / \mathrm{mL}$, 远优于 $\mathrm{Na}$ 盐的溶解度 $(7.5 \mathrm{mg} / \mathrm{mL})$. 此外, 静脉 毒性测试显示 $12 b$ 在 $200 \mathrm{mg} / \mathrm{kg}$ 剂量下不会对小鼠的体 重造成大的改变. 
<smiles>COc1cc2ncnc(Nc3ccc(F)c(Cl)c3)c2cc1OCCCCCCC(=O)NO</smiles>

$12 a$<smiles>C#Cc1cccc(Nc2ncnc3cc(OC)c(OCCCCCCC(=O)NO)cc23)c1</smiles>

$12 b$

2009 年 Reddy 等 ${ }^{[49]}$ 合成了一系列喹唑啉类化合物 13, 在 6-位烷氧基的烷基末端引入咪唑、苯并噻唑等杂 环，生物活性测试结果表明含咪唑基烷氧基的 13a 抑制 $\mathrm{A} 431$ 细胞增殖和 $\mathrm{MCF}-7$ 细胞增殖的 $\mathrm{IC}_{50}$ 值分别为 3.51 和 $38.83 \mu \mathrm{mol} / \mathrm{L}$, 而 $\mathbf{1 3 b}$ 的 $\mathrm{IC}_{50}$ 值则分别为 $3.00,32.65$ $\mu \mathrm{mol} / \mathrm{L}$. 此外, 含苯并噻唑烷氧基且在 4-位芳胺苯环上 有吸电子基团的 $13 \mathrm{c}$ 也具有较好的抗癌活性, 对 A431 和 MCF-7 细胞增殖抑制的 $\mathrm{IC}_{50}$ 值分别为 4.05 和 35.90 $\mu \mathrm{mol} / \mathrm{L}$, 而在 4-位芳胺芳环上引入供电子基团则会降低 其抗癌活性, 如化合物 13d 对 A431 细胞增殖的 $\mathrm{IC}_{50}$ 值 为 $14.17 \mu \mathrm{mol} / \mathrm{L}$. 分子对接结果表明化合物 $\mathbf{1 3 b}$ 与 Gefitinib 作用模式类似, 都与 EGFR 酪氨酸激酶结构域 中的氨基酸残基 Val702, Ala719, Ser696 和 Lys721 相互 作用.<smiles>CCCCc1nc(Cl)c(CCCC)n1CCCOc1cc2c(Nc3cccc(C(F)(F)F)c3)ncnc2cc1OC</smiles>

$13 a$<smiles>CCCc1nc(Cl)c(CCC)n1CCCOc1cc2c(Nc3cccc(I)c3)ncnc2cc1OC</smiles>

$13 b$<smiles>COc1cc2ncnc(Nc3cccc(C(F)(F)F)c3)c2cc1OCCCSc1nc2ccccc2s1</smiles>

$13 c$<smiles>COc1ccc(Nc2ncnc3cc(OC)c(OCCCSc4nc5ccccc5s4)cc23)cc1</smiles>

13d

同年 Reddy 等 ${ }^{[50]}$ 报道了 $13 \mathrm{a}, 13 \mathrm{~b}, 13 \mathrm{e}, 13 \mathrm{f}$ 等对野生 型和突变型 EGFR 的分子相互作用研究, 研究表明 $13 \mathrm{e}$ 抑制 A431 和 MCF-7 细胞增殖的 $\mathrm{IC}_{50}$ 分别为 4.05, 2.24 $\mu \mathrm{mol} / \mathrm{L} ; \mathbf{1 3 f}$ 对 A431 和 MCF-7 细胞的增殖抑制活性 $\mathrm{IC}_{50}$ 值分别为 $4.03,37.26 \mu \mathrm{mol} / \mathrm{L}$. 研究表明含有苯并啞唑的 化合物 $13 \mathrm{e}, 13 \mathrm{f}$ 以及含咪唑侧链的化合物 $13 \mathrm{~b}$ 与野生型 EGFR 结合能最低, 而化合物 13a 对突变型 EGFR, 包括 T790M 突变、L858R 突变以及双突变型 EGFR 的结合 能最低.<smiles>COc1cc2ncnc(Nc3cccc(C(F)(F)F)c3)c2cc1OCCCSc1nc2ccccc2s1</smiles><smiles>COc1ccc(Nc2ncnc3cc(OC)c(OCCCSc4nc5ccccc5s4)cc23)cc1</smiles>

$13 f$

2010 年 Ban 等 ${ }^{[51]}$ 合成了一系列在环 6-位上带有 $\mathrm{C}-\mathrm{C}$ 多重键取代的 4-芳胺喹唑啉化合物, 其中化合物 $14 \mathbf{a} \sim 14 \mathbf{c}$ 抑制 $\mathrm{EGFR}$ 的 $\mathrm{IC}_{50}$ 值分别为 $2.53,3.16$ 和 4.45 $\mathrm{nmol} / \mathrm{L}$, 与对照药物 PD168393 相当 $\left(\mathrm{IC}_{50}\right.$ 值为 2.62 $\mathrm{nmol} / \mathrm{L}$ ), 优于对照药物 Erlotinib, AG1476 和 Gefitinib $\left(\mathrm{IC}_{50}\right.$ 值分别为 $\left.20.3,6.3,21.0 \mathrm{nmol} / \mathrm{L}\right)$; MTT 法检测得到 $14 a$ 和 14b 对 A431 细胞增殖的抑制活性 $\mathrm{GI}_{50}$ 值分别为 $0.221,0.268 \mu \mathrm{mol} / \mathrm{L}$. 研究证实, 在 6 位上带有 C-C 多 重键取代的芳胺喹唑啉的结构对生物活性起关键作用, 含有 $3 \sim 6$ 个碳的长碳链化合物表现出持久的抑制活性.<smiles>C#CCCCOc1cc2c(Nc3ccc(F)c(Cl)c3)ncnc2cc1OC</smiles> 
<smiles>C=C=CCCCOc1cc2c(Nc3ccc(F)c(Cl)c3)ncnc2cc1OC</smiles><smiles>C=C=CCCCCCOc1cc2c(Nc3ccc(F)c(Cl)c3)ncnc2cc1OC</smiles>

2010 年 Chilin 等 ${ }^{[52]}$ 合成了一系列含二氧戊环、二 氧己环、二氧庚环的 4-芳胺基喹唑啉衍生物 15 , 大部分 化合物对 EGF 诱导的 EGFR 磷酸化抑制活性与 PD153035 相当. 化合物 15a 15c 对 A431 细胞增殖抑 制活性 $\mathrm{IC}_{50}$ 值分别为 $0.75,0.67,0.77 \mu \mathrm{mol} / \mathrm{L}$, 活性优于 对照药物 PD153035 ( $\mathrm{IC}_{50}$ 值为 $\left.4.40 \mu \mathrm{mol} / \mathrm{L}\right)$, 且 $\mathbf{1 5 b}$ 和 $15 \mathrm{c}$ 对成纤维细胞 NIH3 3 也有较好的抑制活性, 其 $\mathrm{IC}_{50}$ 值分别为 5.8, 7.1 $\mu \mathrm{mol} / \mathrm{L}$, 而对照药物 PD153035 的 $\mathrm{IC}_{50}$ 值高于 $10 \mu \mathrm{mol} / \mathrm{L}$. 将化合物 $\mathbf{1 5 c}$ 和 $\mathbf{1 5 d}$ 与 EGFR 进行分 子对接实验, 发现 $15 \mathrm{c}$ 能通过 6 位上的氧与 ATP 磷酸化 酶的辅因子镁离子相互作用, 与 EGFR 结合良好, 从而 抑制活性高于 15d(抑制 A431 细胞和 NIH3T3 的 $\mathrm{IC}_{50}$ 值 分别为 $8.46>10 \mu \mathrm{mol} / \mathrm{L})$. 上述结果说明在这一系列化 合物结构中二氧烷环在生物活性中起关键作用, 尤其是 含二氧已环的喹唑啉结构对于设计高活性的 EGFR 抑制 剂更具有指导性.

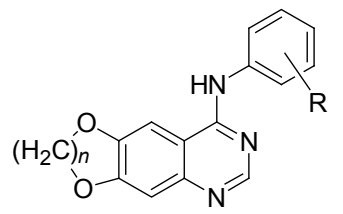

15a $n=2, \mathrm{R}=3-\mathrm{Br} ; 15 \mathrm{~b} n=2, \mathrm{R}=3-\mathrm{Me} ; 15 \mathrm{c} n=2, \mathrm{R}=3-\mathrm{CF}_{3} ; 15 \mathrm{~d}$ $n=1, \mathrm{R}=3-\mathrm{CF}_{3}$

2011 年 Garofalo 等 ${ }^{[53]}$ 合成了一系列 4-位苯环上带 有氨基甲酸酯取代基的化合物 16, 测试其对 EGFR 和抗 PC3, HT29, MCF-7 和 EAHY926 细胞增殖的活性. 研究 发现在 4-苯胺的苯环上氨基甲酸酯基团的邻位引入 $\mathrm{Cl}$ 或 Me 时, 化合物具有同时抑制 EGFR 和 VEGFR 的双 重功能. 如活性较好的化合物 16a, 16b, 抑制 EGFR 的 $\mathrm{IC}_{50}$ 值分别为 1.0 和 $0.9 \mu \mathrm{mol} / \mathrm{L}$, 抑制 $\mathrm{VEGFR}$ 的 $\mathrm{IC}_{50}$ 值 分别为 0.5 和 $0.85 \mu \mathrm{mol} / \mathrm{L}$, 与对照药物 ZD6476(抑制 EGFR 和 VEGFR 的 $\mathrm{IC}_{50}$ 值分别为 0.8 和 $0.1 \mu \mathrm{mol} / \mathrm{L}$ ) 的 活性相当. 抑制癌细胞增殖活性试验发现，化合物 16 在
$10 \mu \mathrm{mol} / \mathrm{L}$ 浓度下对上述癌细胞的抑制活性很低或几乎 无抑制活性. 进一步对喹唑啉环上的 6-位或 7-位进行修 饰，发现喹唑啉环 6,7-位上引入胺基烷基取代可提高抑 制癌细胞增殖活性，如化合物 16c 抑制 HT29, MCF-7 和 EAHY926 的 $\mathrm{IC}_{50}$ 值分别为 $2.5,7.1$ 和 $2.8 \mu \mathrm{mol} / \mathrm{L}$, 对 HT29 和 EAHY926 的抑制活性优于对照药剂 ZD6476 (Vandetanib, 抑制 HT29 和 EAHY926 的 $\mathrm{IC}_{50}$ 值分别为 4.2, $5.1 \mu \mathrm{mol} / \mathrm{L})$.<smiles>CCOC(=O)Nc1ccc(Nc2ncnc3cc(OC)c(OC)cc23)cc1Cl</smiles><smiles>CCCOC(=O)Nc1ccc(Nc2ncnc3cc(OC)c(OC)cc23)cc1C</smiles><smiles>CCCCOc1cc2c(Nc3ccc(NC(=O)OCC)c(Cl)c3)ncnc2cc1OCCNCC</smiles><smiles>COc1cc2c(Nc3ccc(Br)cc3F)ncnc2cc1OCC1CCN(C)CC1</smiles>

\subsection{6-胺基-7-烷氧基-4-芳胺喹唑啉}

2007 年 Ahn 等 ${ }^{[54]}$ 合成了 173 个 6-丙烯酰胺基-4-芳 胺喹唑啉化合物 17, 测试了其抑制 EGFR 和 A431 细胞 生长的活性，结果表明其中 135 个化合物有良好的抑制 EGFR 和 A431 细胞增殖的生物活性, 其 $\mathrm{IC}_{50}$ 值范围为 3 98 nmol/L. 在该系列化合物中 $\mathbf{1 7} \mathbf{a}$ 和 $17 \mathbf{b}$ 的抑制活 性最好, 17a, 17b 抑制 EGFR 的 $\mathrm{IC}_{50}$ 值分别为 6,7 $\mathrm{nmol} / \mathrm{L}$, 抑制 $\mathrm{A} 431$ 细胞增殖的 $\mathrm{IC}_{50}$ 值均为 $8 \mathrm{nmol} / \mathrm{L}$. 


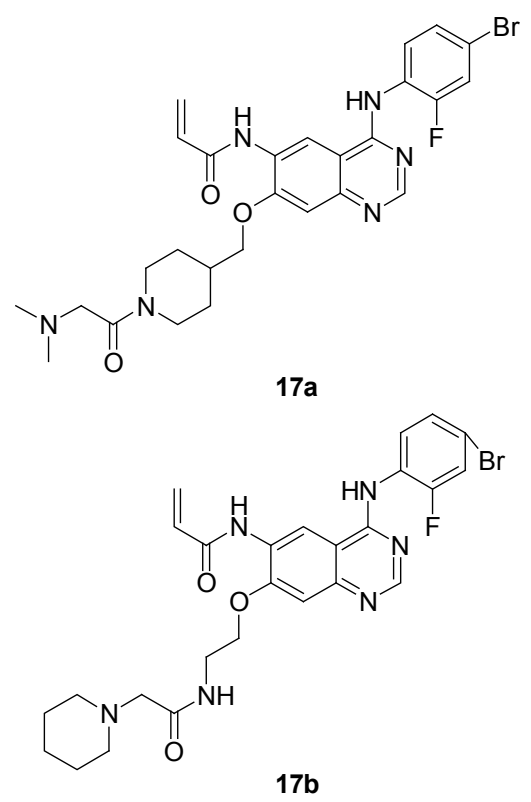

2007 年 Dissoki 等 ${ }^{[55]}$ 合成了 4 个含 ${ }^{18} \mathrm{~F}$ 的 PEG 取代 的喹唑啉化合物 $18 \mathrm{a} \sim 18 \mathrm{~d}$, 发现化合物对 A431 细胞 EGFR 自磷酸化的抑制活性 $\mathrm{IC}_{50}$ 值为 $5 \sim 40 \mathrm{nmol} / \mathrm{L}$. 其 中 $18 \mathrm{a} \sim 18 \mathrm{c}$ 为可逆抑制剂, 而活性最好的化合物 $18 \mathrm{~d}$ $\left(\mathrm{IC}_{50}\right.$ 值为 $\left.5 \mathrm{nmol} / \mathrm{L}\right)$ 为不可逆抑制剂. 且 $\mathbf{1 8 d}$ 的水溶性 (溶解度为 $3.5 \mu \mathrm{g} / \mathrm{mL}$ ) 远超过 18a $\sim 18 \mathbf{c}$ (溶解度为 $0.009 \sim$ $0.03 \mu \mathrm{g} / \mathrm{mL}$ ), 具有很好的研究开发前景.

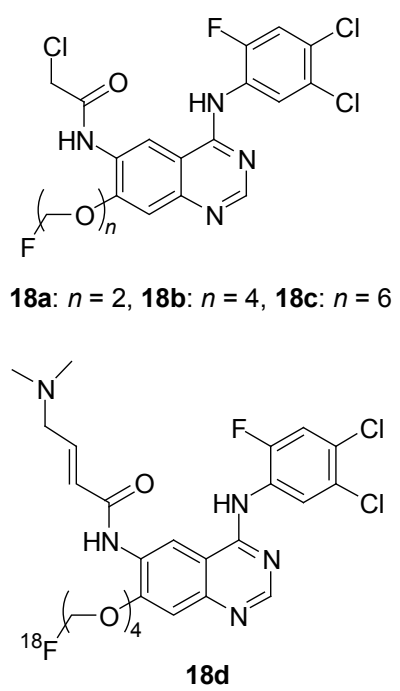

2007 年 Wissner 等 ${ }^{[56]}$ 合成了一系列 7-乙氧基-6-丁 烯酰胺基基取代-4- $N$-取代喹唑啉化合物 19, 发现该系 列化合物均有一定的 EGFR 抑制活性, 其中化合物 19a 的抑制活性最好, 作为不可逆抑制剂, 该化合物喹唑啉 环上 6-(4-二甲胺基)丁烯酰胺基作为迈克尔受体与 EGFR 的 cys-773 氨基酸残基结合, 在 $1 \mu \mathrm{mol} / \mathrm{L}$ 的 ATP 浓度下 $\mathrm{IC}_{50}$ 值为 4.1 , 在 $1 \mathrm{mmol} / \mathrm{L}$ ATP 的浓度下 $\mathrm{IC}_{50}$ 值 为 $43.1 \mathrm{nmol} / \mathrm{L}$, 而对照药物 Gefitinib 在 $1 \mu \mathrm{mol} / \mathrm{L}$ 和 1 $\mathrm{mmol} / \mathrm{L}$ 的 ATP 浓度下抑制 $\mathrm{EGFR}$ 活性的 $\mathrm{IC}_{50}$ 值分别为 5.4 和 $2753 \mathrm{nmol} / \mathrm{L}$.<smiles>CCOc1cc2ncnc(NC3=CC(=O)C(OCc4cccc(F)c4)C=C3C(=O)O)c2cc1NC(=O)/C=C/CN(C)C</smiles>

2008 年 $\mathrm{Li}$ 等 ${ }^{[57]}$ 报道了 BIBW2992 作为 EGFR 的不 可逆抑制剂对潜伏期肺癌具有高活性. 该化合物为 2002 年在世界专利 WO 02/50043 中报道合成 ${ }^{[58]}$. 体外 酶活性测试结果表明 BIBW2992 对野生型和突变型 EGFR 均有很好的抑制活性, 对 WT-EGFR 的 $\mathrm{IC}_{50}$ 值为 $0.5 \mathrm{nmol} / \mathrm{L}$, 优于对照药物 Gefitinib 的 $\mathrm{IC}_{50}$ 值 $3 \mathrm{nmol} / \mathrm{L}$, 而对突变型 LR-EGFR 的抑制 $\mathrm{IC}_{50}$ 值为 $0.4 \mathrm{nmol} / \mathrm{L}$, 与 Gefitinib 的 $\mathrm{IC}_{50}$ 值 $0.8 \mathrm{nmol} / \mathrm{L}$ 相当; 但对已经对 Gefitinib 产生抗性的 LR/TM-EGFR 双突变型, 其抑制 $\mathrm{IC}_{50}$ 值为 $10 \mathrm{nmol} / \mathrm{L}$, 远优于对照药物 Gefitinib 的 $\mathrm{IC}_{50}$ 值为 1013 $\mathrm{nmol} / \mathrm{L}$. 以 Erlotinib 为对照药物, 测试了 BIBW2992 抑 制细胞 EGFR 自磷酸化的活性, 结果表明 BIBW2992 在 $10 \mathrm{nmol} / \mathrm{L}$ 可明显抑制 LR-EGFR 的磷酸化, 在 100 $\mathrm{nmol} / \mathrm{L}$ 可抑制 LR/TM-EGFR 的磷酸化. 进一步测试了 该化合物抑制各种肺癌细胞增殖的活性，证实该化合物 对表达 WT-EGFR 或 LR-EGFR、LR/TM-EGFR 的肺癌 细胞的增殖抑制活性 $\mathrm{IC}_{50}$ 值, 分别为 $60,0.7,99 \mathrm{nmol} / \mathrm{L}$, 而对照药物 Gefitinib 的 $\mathrm{IC}_{50}$ 值分别为 $157,5,>4000$ $\mathrm{nmol} / \mathrm{L}$, Erlotinib 的 $\mathrm{IC}_{50}$ 值分别为 $110,40,>4000$ $\mathrm{nmol} / \mathrm{L}$. 在临床前活体抑瘤试验中, 口服剂量 $20 \mathrm{mg}$ • $\mathrm{kg}^{-1} \cdot \mathrm{d}^{-1}$ 下, 连续服用 $25 \mathrm{~d}$ 的肿瘤体积缩小率治疗组与 对照组相比 $(\mathrm{T} / \mathrm{C})$ 为 $2 \%$, 效果优于 Gefitinib(口服剂量为 $75 \mathrm{mg} \cdot \mathrm{kg}^{-1} \cdot \mathrm{d}^{-1}, \mathrm{~T} / \mathrm{C}=46 \%$ ); 对表达 $\mathrm{LR} / \mathrm{TM}$ 双突变型 EGFR 的 NCIH1975 细胞所导致的肿瘤有抑制活性, 体 积治疗率为 $12 \%$ (治疗组比对照组, $\mathrm{T} / \mathrm{C}$ ), 尤其是对已经 对 Erlotinib 产生抗性的原发小鼠肺癌也有很好的治疗活 性，每日口服剂量 $20 \mathrm{mg} \cdot \mathrm{kg}^{-1} \cdot \mathrm{d}^{-1}$ 下，治疗 4 周后，肿瘤 减小了 50\%. 目前 BIBW2992 的一期临床试验已经结 束, 已进入二期临床试验阶段 ${ }^{[59]}$.

\section{3 其他 6-取代-7-烷氧基-4-芳胺喹唑啉}

2005 年 Hennequin 和 Halsall ${ }^{[60]}$ 报道了一系列喹唑啉 化合物 20, 该系列化合物抑制 $\mathrm{KB}$ 细胞 $\mathrm{EGFR}$ 的 $\mathrm{IC}_{50}$ 值 在 $0.001 \sim 10 \mu \mathrm{mol} / \mathrm{L}$ 之间, 其中化合物 $20 \mathrm{a} \sim 20 \mathrm{~d}$ 对 EGFR 酪氨酸磷酸的 $\mathrm{IC}_{50}$ 值分别为 $64,20,67,52 \mathrm{nmol} / \mathrm{L}$ 
而对 $\mathrm{EGFR}$ 诱导的 $\mathrm{KB}$ 细胞增殖的 $\mathrm{IC}_{50}$ 值分别为 143,50 , $160,290 \mathrm{nmol} / \mathrm{L}$ ，具有进一步开发的潜力.<smiles>COc1cc2ncnc(Nc3ccc(F)c(Cl)c3)c2cc1CN1CCC[C@H]1C(=O)NCC#N</smiles>

$20 \mathrm{a}$<smiles>COc1cc2ncnc(Nc3cccc(Cl)c3F)c2cc1CN1CSC[C@H]1C(N)=O</smiles>

$20 b$<smiles>COCCN1CCN(c2cc3c(Nc4cccc(Cl)c4F)ncnc3cc2OC)C(C(N)=O)C1</smiles><smiles>COc1cc2ncnc(Nc3cccc(Cl)c3F)c2cc1CC1CC(C(N)=O)C1</smiles>

$20 \mathrm{c}$

2005 年 Delouvrie 等 ${ }^{[61]}$ 报道了一系列 7-位酰胺基 亚甲基取代的喹唑啉化合物 21，该系列化合物中 21a 21d 对 EGFR 蛋白质磷酸化的 $\mathrm{IC}_{50}$ 值分别为 $17,17,27,9$ $\mathrm{nmol} / \mathrm{L}$, 而对 $\mathrm{EGFR}$ 诱导的 $\mathrm{KB}$ 细胞增殖的 $\mathrm{IC}_{50}$ 值分别 为 $23,36,54,91 \mathrm{nmol} / \mathrm{L}$.<smiles></smiles><smiles>[Y]C(CO)(C(N)=O)C(=O)NC</smiles>

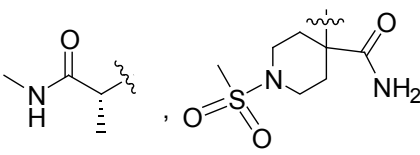

\section{6-取代-4-芳胺喹唑啉}

\subsection{6 -胺基-4-芳胺喹唑啉}

2001 年 Tsou 等 ${ }^{[62]}$ 合成了一系列对 EGFR 和 HER-2 具有不可逆抑制活性的 6-取代-4-(3-溴苯胺)喹唑啉衍生 物 22. 该系列化合物抑制 $\mathrm{EGFR}$ 的 $\mathrm{IC}_{50}$ 值为 $0.002 \sim$ $1.124 \mu \mathrm{mol} / \mathrm{L}$ ，抑制 HER-2 的 $\mathrm{IC}_{50}$ 值为 $0.032 \sim 91.325$ $\mu \mathrm{mol} / \mathrm{L}$ ，抑制 $\mathrm{A} 431$ 细胞的 $\mathrm{IC}_{50}$ 值为 $0.016 \sim 8.500$ $\mu \mathrm{mol} / \mathrm{L}$, 抑制 $\mathrm{SKBR} 3$ 细胞的 $\mathrm{IC}_{50}$ 值为 $0.001 \sim 2.220$ $\mu \mathrm{mol} / \mathrm{L}$ ，抑制 SW620 细胞的 $\mathrm{IC}_{50}$ 值为 $0.198 \sim 20.700$ $\mu \mathrm{mol} / \mathrm{L}$. 开展了活性较高的化合物 $22 \mathrm{a}$ 的裸鼠肿瘤抑制 活体试验，结果表明在口服剂量 $40 \mathrm{mg} \cdot \mathrm{kg}^{-1} \cdot \mathrm{d}^{-1}$ 下, $10 \mathrm{~d}$ 后对 A431 细胞导致的肿瘤的抑制率超过 $90 \%$.<smiles>CN(C)C/C=C/C(=O)Nc1ccc2ncnc(Nc3cccc(Br)c3)c2c1</smiles>

22a

2004 年 Qiu 等 ${ }^{[63]}$ 报道了 5-亚硝基脲的喹唑啉化合 物 FD137，该化合物和其代谢产物 FD110 可抑制 DU145 人前列腺癌细胞中 EGFR 的磷酸化和阻断 c-fos 基因的 表达，且具有剂量效应, FD110 在 $30 \mu \mathrm{mol} / \mathrm{L}$ 浓度下可完 全抑制 EGFR 的自磷酸化. 此外, 还发现 FD137 可诱导 DNA 损伤且其对 DU145 的增殖抑制活性高出对照亚硝 基脲类化疗药物卡莫司汀(BCNU)对 DU145 细胞增殖抑 制活性的 150 倍. 传统上亚硝基脲类药物对前列腺癌并 无明显治疗活性，而FD137 优异的活性可能是由于它同 时以 DNA 和 EGFR 及其下游信息转导为靶标, 这种组 合靶标方法意味着对 EGFR过度表达的前列腺癌可以采 用 $N$-亚硝基腿类药物进行治疗的新策略.<smiles>Cc1cccc(Nc2ncnc3ccc(N(C)C(=O)N(CCCl)N=O)cc23)c1</smiles>

FD137<smiles>CNc1ccc2ncnc(Nc3cccc(C)c3)c2c1</smiles>

FD110
2004 年 Mishani 等 ${ }^{[64]}$ 在前期合成的可逆抑制剂 4-(3,4-二氯-6-氟苯胺)-6,7-二甲氧基喹唑啉(ML01)和 6丙烯酰胺-4-(3,4-二氯-6-氟苯胺)喹唑啉(ML03) ${ }^{[65]}$ 的基 础上，设计合成 ${ }^{11} \mathrm{C}$ 标记的 $23 \mathbf{a} \sim 23 \mathrm{c}$ 作为显像剂，发现 化学稳定性更好的 23a 23c 不仅可作为 EGFR 高度表 达癌细胞的分子显像剂，同时具有良好的不可逆抑制 EGFR 自磷酸化的作用，具体活性数据见表 1 . 
表 1 酶联免疫法测定化合物对 A431 细胞裂解液中 EGFR 的 $\mathrm{IC}_{50}$ 值、和对未受损伤的 $\mathrm{A} 431$ 细胞在移去含化合物的培养基 后立即测试及 $8 \mathrm{~h}$ 后测试所得的 EGFR 自磷酸化的 $\mathrm{IC}_{80}$ 值 ${ }^{a}$

Table $1 \mathrm{IC}_{50}$ values in the ELISA screen with A431 cell lysates and $\mathrm{IC}_{80}$ values for inhibition of EGFR autophosphorylation in intact A431 cells immediately after and $8 \mathrm{~h}$ post removal of the compound from the medium

\begin{tabular}{|c|c|c|c|}
\hline \multirow{2}{*}{ Compd. } & \multirow{2}{*}{$\begin{array}{l}\text { 对 } \mathrm{A} 431 \text { 裂解液 } \\
\mathrm{IC}_{50} /\left(\mathrm{nmol} \cdot \mathrm{L}^{-1}\right)\end{array}$} & \multicolumn{2}{|c|}{$\begin{array}{c}\text { 对未受损伤 } \mathrm{A} 431 \text { 细胞 } \\
\mathrm{IC}_{80} /\left(\mathrm{nmol} \cdot \mathrm{L}^{-1}\right)\end{array}$} \\
\hline & & $\begin{array}{c}\text { 除去抑制剂 } \\
\text { 后的瞬间 }\end{array}$ & $\begin{array}{c}\text { 除去抑制剂的 } \\
8 \mathrm{~h} \text { 后 }\end{array}$ \\
\hline ML01 & 0.208 & $3 \sim 5$ & - \\
\hline ML03 & 0.037 & $6.7 \sim 20$ & $6.7 \sim 20$ \\
\hline 23a & $0.06 \pm 0.04$ & $4 \sim 10$ & $10 \sim 50$ \\
\hline $23 b$ & $0.46 \pm 0.12$ & $4 \sim 10$ & $\approx 50$ \\
\hline $23 \mathrm{c}$ & $0.09 \pm 0.01$ & $10 \sim 20$ & $10 \sim 20$ \\
\hline
\end{tabular}

${ }^{a} \mathrm{IC}_{50}$ 值是使用 ELISA 方法测试化合物对 $\mathrm{A} 431$ 细胞裂解液的 EGFR 磷酸 化的抑制活性达 $50 \%$ 的浓度, $\mathrm{IC}_{80}$ 值是化合物对未受损伤的 $\mathrm{A} 431$ 细胞中 EGFR 自磷酸化抑制活性达 $80 \%$ 的浓度.<smiles>[X]c1ccc(Nc2ncnc3ccc(NC(=O)/C=C/CN(C)[14CH3])cc23)cc1</smiles>

23a $\mathrm{X}=3,4-\mathrm{Cl}_{2}-6-\mathrm{F}, \mathbf{2 3 b} \mathrm{X}=m-\mathrm{Br}, \mathbf{2 3 c} \mathrm{X}=m-\mathrm{I}$

2004 年 Matheson 等 ${ }^{[66]}$ 报道了化合物 SMA41 在体 外和活体中的抗癌活性. SMA41 通过不可逆机理阻断 EGF 诱导的 EGFR 自磷酸化, 在 $25 \mu \mathrm{mol} / \mathrm{L}$ 浓度下, SMA41 抑制 EGFR 的自磷酸化效果达到 $90 \%$. 此外, SMA41 对 A431 细胞中的丝裂原活化蛋白激酶(MAPK) 的活性具有很好的抑制作用. 活体试验表明 SMA41 生 物耐受性良好, 在 $200 \mathrm{mg} \cdot \mathrm{kg}^{-1} \cdot \mathrm{d}^{-1}$ 剂量下, 在植入 A431 细胞的免疫系统去除的 SCID 鼠中, SMA41 对肿瘤 的增殖抑制活性是母体化合物 SMA52 的两倍, 这同样 是由于化合物 SMA41 兼具抑制 EGFR 磷酸化以及诱导 DNA 损伤两种功能.<smiles>CNNNc1ccc2ncnc(Nc3cccc(C)c3)c2c1</smiles><smiles>Cc1cccc(Nc2ncnc3ccc(N)cc23)c1</smiles>

2005 年 Brahimi 等 ${ }^{[67]}$ 报道了以 SMA41 为母体设计 合成的喹唑啉化合物 ZRBA1, 该化合物在喹唑啉 6-位
上含有 3-( N,N-二甲胺基乙基)-三氮脒基团, 既能增加水 溶性，且具有烷基化活性，对 EGFR 的 $\mathrm{IC}_{50}$ 值为 37 $\mathrm{nmol} / \mathrm{L}$, 抑制活性超过母体化合物 5 倍. 水解后生成 $\mathrm{FD} 105\left(\mathrm{IC}_{50}\right.$ 值为 $\left.200 \mathrm{nmol} / \mathrm{L}\right), \mathrm{ZRBA} 1$ 对 EGFR-TK ATP 位点的亲和力比 FD105 高 5 倍. 此外, ZRBA1 可诱导 DNA 损伤, 在 $1 \mu \mathrm{mol} / \mathrm{L}$ 浓度下, 对 MDA-MB-468 乳腺 癌细胞中 EGFR 的磷酸化的不可逆抑制活性达 $98 \%$. 与 FD105 和 SMA41 相比, ZRBA1 可以阻断 EGFR 下游的 MAPK 的活性, 诱导更多细胞的调亡. 对植入有乳腺癌 细胞的 SCID 小鼠的活体试验表明, 化合物 ZRBA1 在口 服剂量 $50 \mathrm{mg} \cdot \mathrm{kg}^{-1} \cdot \mathrm{d}^{-1}$ 下比 SMA41 抑制活性更高.<smiles>CN(C)CCN=NNc1ccc2ncnc(Nc3cccc(Cl)c3)c2c1</smiles>

ZRBA1<smiles>Nc1ccc2ncnc(Nc3cccc(Cl)c3)c2c1</smiles>

FD105
2006 年 Domarkas 等 ${ }^{[68]}$ 以 EGFR 为靶标合成了一系 列稳定的 $N$-亚硝基脲类喹唑啉类化合物 $\mathbf{2 4}$, 并与氨基 喹唑啉类化合物进行了对比. 研究发现氨基喹唑啉类化 合物 $24 \mathrm{a}$ 和 $24 \mathrm{~b}$ 对 $\mathrm{EGFR}$ 的 $\mathrm{IC}_{50}$ 值分别为 4 和 $5 \mathrm{nmol} / \mathrm{L}$, 而化合物 $\mathbf{2 4 c}$ 的 $\mathrm{IC}_{50}$ 值为 $1.139 \mu \mathrm{mol} / \mathrm{L}$, 活性远低于 $\mathbf{2 4 a}$ 和 24b. 研究发现, 化合物 24c 的亚硝基腿平面与喹唑 啉环平面扭转角较大, 达为 $17.65^{\circ}$ 的化合物, 对 EGFR 狭窄的 ATP 竞争裂口有很强的位阻作用. 但是由于亚 硝基腿基团的存在，化合物 $\mathbf{2 4 a} \sim \mathbf{2 4 c}$ 对 $\mathrm{NIH} 3 \mathrm{~T} 3$ 的增殖 抑制活性分别为 $49.8,17.6,16.5 \mu \mathrm{mol} / \mathrm{L}, \mathbf{2 4} \mathbf{c}$ 显现出了最 高的增殖抑制活性，原因同样在于化合物除具有 EGFR 抑制活性外，还有 DNA 损伤作用.<smiles>CNc1ccc2ncnc(Nc3cccc(Cl)c3)c2c1</smiles><smiles>[Z14][X]</smiles>

24b<smiles></smiles>

24c 
2006 年 Ham 等 ${ }^{[69}$ 报道了一系列 4-吡啶亚甲氧基苯 胺基-6-酰胺基喹唑啉化合物 25, 测试了该系列化合物 抑制 A431, SK-Br-3, SW-620 细胞增殖和 EGFR 的生物 活性，结果表明共有 21 个化合物对 EGFR 以及 SK-Br-3 细胞增殖具有较高的抑制活性, 其 $\mathrm{IC}_{50}$ 值分别为 $2 \sim 4.7$ 和 $1 \sim 5 \mathrm{nmol} / \mathrm{L}$, 活性远优于对照药物 Gefitinib $\left(\mathrm{IC}_{50}\right.$ 值 分别为 0.206 和 $1.47 \mu \mathrm{mol} / \mathrm{L})$ 和 Erlotinib $\left(\mathrm{IC}_{50}\right.$ 值为 0.891 和 $>1.7 \mu \mathrm{mol} / \mathrm{L})$, 其中有 14 个化合物有良好的抑制 $\mathrm{A} 431$ 细胞增殖的活性，其 $\mathrm{IC}_{50}$ 值为 $0.010 \sim 0.027$ $\mu \mathrm{mol} / \mathrm{L}$, 优于对照药物 Gefitinib $\left(\mathrm{IC}_{50}\right.$ 值为 $\left.0.028 \mu \mathrm{mol} / \mathrm{L}\right)$ 和 Erlotinib $\left(\mathrm{IC}_{50}\right.$ 值为 $\left.0.059 \mu \mathrm{mol} / \mathrm{L}\right)$, 化合物 $25 \mathrm{a}$ 的活性 最好, 抑制 $\mathrm{A} 431, \mathrm{SK}-\mathrm{Br}-3$ 细胞和 $\mathrm{EGFR}$ 的 $\mathrm{IC}_{50}$ 值分别 为 9, 2 和 $1 \mathrm{nmol} / \mathrm{L}$, 而对不过度表达 EGFR 的 SW-620 细胞并没有抑制活性，说明该系列化合物对由于 EGFR 过度表达导致增殖的癌细胞具有很好的选择抑制活性, 有进一步开发成治疗癌症药物的可能.<smiles>C=CC(=O)N(C)CCC(=O)Nc1ccc2ncnc(Nc3ccc(OCc4ccccn4)c(Cl)c3)c2c1</smiles>

2007 年 Fernandes 等 ${ }^{[70]}$ 报道了以 6-胺基-4- $N$-(3-氯4-氟苯胺)喹唑啉为母体合成的两个化合物 $26 \mathbf{a}$ 和 $26 \mathbf{b}$. MTT 法表明，化合物 26a 和放射性碘标记的化合物 $\left.{ }^{125} \mathrm{I}\right]-26 \mathrm{~b}\left(\mathrm{IC}_{50}\right.$ 值分别为 $3 \sim 12$ 和 $\left.2 \sim 7 \mu \mathrm{mol} / \mathrm{L}\right)$ 比它们的 母体化合物 6-胺基-4- $N$-(3-氯-4-氟苯胺)喹唑啉 $\left(\mathrm{IC}_{50}\right.$ 值 为 $25 \sim 30 \mu \mathrm{mol} / \mathrm{L}$ ) 具有更好的抑制 A431 细胞增殖的活 性，且这两个化合物在 $0.1 \mu \mathrm{mol} / \mathrm{L}$ 浓度下能完全抑制 EGFR 自磷酸化<smiles>Nc1ccc2ncnc(Nc3ccc(F)c(Cl)c3)c2c1</smiles>

6-胺基-4- $N-(3-$ 氯-4-氟苯胺)喹唑啉<smiles>O=C(CCBr)Nc1ccc2ncnc(Nc3ccc(F)c(Cl)c3)c2c1</smiles><smiles>O=C(CCI)Nc1ccc2ncnc(Nc3ccc(F)c(Cl)c3)c2c1</smiles>

26b

2007 年 Blair 等 ${ }^{[71]}$ 设计合成了荧光亲合探针化合物 27, 可以对 EGF 诱导的 EGFR 磷酸化和它的下游信号途 径效应分子 Akt, Erk1/2 磷酸化进行量化.<smiles>C=CC(=O)Nc1cc2c(Nc3cccc(Br)c3)ncnc2cc1OCCOCCOCCOCCNC1CC=C([N+](=O)[O-])c2nonc21</smiles>

2008 年 Michalczyk 等 ${ }^{[72]}$ 合成了 6-丙烯酰胺取代的 喹唑啉化合物 $28 \mathrm{a} \sim 28 \mathrm{c}$, 测试了化合物 $28 \mathrm{a}, \mathbf{2 8 b}$ 以及 28c 对 EGFR 和 EGFR-T790M 的抑制活性. 28a, 28b 对 EGFR 具有不可逆抑制活性，其 $\mathrm{IC}_{50}$ 值分别为 $0.08,0.22$ $\mathrm{nmol} / \mathrm{L}$, 而 28c 对 EGFR 具有可逆抑制活性, 其 $\mathrm{IC}_{50}$ 值 为 $0.43 \mathrm{nmol} / \mathrm{L}$, 均优于对照药物 Erlotinib $\left(\mathrm{IC}_{50}\right.$ 值为 3.6 $\mathrm{nmol} / \mathrm{L})$; 三个化合物对 EGFR-T790M 突变型的抑制活 性 $\mathrm{IC}_{50}$ 值分别为 $1.2,3.0,360 \mathrm{nmol} / \mathrm{L}$ ，可看出 $\mathbf{2 8 a}$ 和 $\mathbf{2 8 b}$ 对突变型 EGFR 的抑制活性远优于对照药物 Erlotinib $\left(\mathrm{IC}_{50}\right.$ 值为 $\left.260 \mathrm{nmol} / \mathrm{L}\right)$, 说明不可逆抑制剂能在一定程 度上克服 EGFR 突变所导致的药物抗性.<smiles>C=CC(=O)Nc1ccc2ncnc(Nc3cccc(Br)c3)c2c1</smiles><smiles>CN(C)C/C=C/C(=O)Nc1ccc(N)c(C(=N)Nc2cccc(Br)c2)c1</smiles><smiles>CCC(=O)Nc1ccc2ncnc(Nc3cccc(Br)c3)c2c1</smiles>

28c

2009 年 Paravatou-Petsotas 等 ${ }^{[73]}$ 为发展针对 EGFR 的生物标记物，在 4-间溴苯胺喹唑啉的 6 位上进行修饰 得到 6-吡啶亚胺基喹唑啉类化合物 29a，并进一步合成 
了 29a 的铼配位化合物 29b, 离体生物活性测试结果表 明化合物 29a, 29b 在未受损伤的 A431 细胞中可逆性抑 制 EGFR 磷酸化, 其 $\mathrm{IC}_{50}$ 值分别为 $(17 \pm 3.7)$ 和(114 23 ) $\mathrm{nmol} / \mathrm{L}$, 而对 $\mathrm{A} 431$ 细胞增殖抑制活性 $\mathrm{IC}_{50}$ 值分别为 $(5.2 \pm 1.1)$ 和 $(2.0 \pm 0.98) \mu \mathrm{mol} / \mathrm{L}$.<smiles>Brc1cccc(Nc2ncnc3ccc(/N=C/c4ccccn4)cc23)c1</smiles><smiles>[X]C(c1ccc2ncnc(Nc3cccc(Br)c3)c2c1)([N+](=O)[O-])[N+]([O-])([O-])[O-]</smiles>

2009 年 Rachid 等 ${ }^{[74]}$ 为了增强三氮脒类化合物的稳 定性, 以 RB107 为先导, 对 6 位上的三氮脒的乙酰氧基 取代基改造成烷氧羰基取代, 设计合成了 6 个新的喹唑 啉类化合物 30, 以 Gefitinib(抑制 EGFR 磷酸化的 $\mathrm{IC}_{50}$ 值为 $0.033 \mu \mathrm{mol} / \mathrm{L}$, 抑制 NIH3T3 细胞增殖的 $\mathrm{IC}_{50}$ 值为 $0.54 \mu \mathrm{mol} / \mathrm{L}$ ) 和 $\mathrm{RB} 107^{[75,76]}$ (抑制 EGFR 磷酸化的 $\mathrm{IC}_{50}$ 值 为 $0.24 \mu \mathrm{mol} / \mathrm{L}$ ) 为对照药物, 测得其抑制 EGFR 磷酸化 的 $\mathrm{IC}_{50}$ 值范围为 $0.18 \sim 0.77 \mu \mathrm{mol} / \mathrm{L}$, 抑制 $\mathrm{NIH} 3 \mathrm{~T} 3$ 细胞 增殖的 $\mathrm{IC}_{50}$ 值范围为 $1.50 \sim 4.94 \mu \mathrm{mol} / \mathrm{L}$. 尽管该系列中 活性最好的化合物 30a 对 NH3T3 细胞增殖抑制活性仍 弱于 Gefitinib, 但是与 RB107 比较, 稳定性增加, 抑制 活性却没有减弱. 证明将三氮脒 N-3 位上的羧甲基换成 氨基甲酸盐可以增强化合物的稳定性而不会影响其抑 制活性.<smiles>CC(=O)OCN(C)N=Nc1ccc2ncnc(Nc3cccc(Cl)c3)c2c1</smiles>

RB107<smiles>CC(=O)OCOC(=O)N(C)N=Nc1ccc2ncnc(Nc3cccc(Cl)c3)c2c1</smiles>

$30 \mathrm{a}$

\subsection{6 -烷氧基-4-芳胺喹唑啉}

2005 年 Bradbury ${ }^{[77]}$ 报道了一系列 6-烷氧基-4-杂环 亚甲氧基苯胺喹唑啉化合物 31, 该系列化合物对 $\mathrm{KB}$ 细 胞中 $\mathrm{EGFR}$ 磷酸化抑制活性 $\mathrm{IC}_{50}$ 值范围为 $0.001 \sim 5$ $\mu \mathrm{mol} / \mathrm{L}$ ，活体试验表明适合的口服剂量为 $1 \sim 200 \mathrm{mg} \cdot$ $\mathrm{kg}^{-1} \cdot \mathrm{d}^{-1}$. 其中化合物 31a 31c 对 EGFR 酪氨酸激酶磷 酸化的抑制活性 $\mathrm{IC}_{50}$ 值分别为 $0.039,0.021,0.213$ $\mu \mathrm{mol} / \mathrm{L}$ ，具有良好的进一步开发价值.<smiles>[R]Oc1ccc2ncnc(Nc3ccc(O[R])c(Cl)c3)c2c1</smiles>

a:<smiles>[R]CN(C)CC(=O)N1CCC([AlH])CC1</smiles><smiles>[R]N(C)CC(=O)N1CCC([I-])CC1</smiles><smiles>[R]C(C)(C)Cc1cncnc1</smiles><smiles></smiles><smiles>[Al]Cc1ccc[R]n1</smiles>

2009 年 Ban 等 ${ }^{[78]}$ 合成了 6 -硼取代烷氧基-4-苯胺基 喹唑啉类化合物 $32 \mathrm{a} \sim 32 \mathrm{c}$, 该类化合物在体外具有较 好的抑制 $\mathrm{EGFR}$ 的生物活性, 其 $\mathrm{IC}_{50}$ 值为 $0.20 \sim 0.85$ $\mu \mathrm{mol} / \mathrm{L}$ ，其中活性最高的化合物 32a 对 EGFR 酪氨酸激 酶抑制活性的 $\mathrm{IC}_{50}$ 值为 $0.19 \mu \mathrm{mol} / \mathrm{L}$. 进一步的分子对接 模拟研究表明化合物 32c 的硼原子和 EGFR 的 Asp800 形成了 $\mathrm{B}-\mathrm{O}$ 共价相互作用，从而使化合物具有长效抑 制 EGFR 磷酸化活性，使化合物 32 也具有了不可逆抑制 剂的特性. 化合物 32a 32c 对 $\mathrm{A} 431$ 细胞增殖的 $\mathrm{GI}_{50}$ 值 为 $0.53 \sim 2.42 \mu \mathrm{mol} / \mathrm{L}$.<smiles>CC1(C)C2CC3OB(COc4ccc5ncnc(Nc6cccc(Cl)c6)c5c4)OC3(C)C1C2</smiles>

$32 a$<smiles>OB(O)/C=C/COc1ccc2ncnc(Nc3cccc(Cl)c3)c2c1</smiles>

$32 b$<smiles>OB(O)c1ccc(COc2ccc3ncnc(Nc4cccc(Cl)c4)c3c2)cc1</smiles>

32c 


\section{3 其它 6-取代-4-芳胺喹唑啉}

2001 年 Rusnak 等 ${ }^{[79]}$ 报道了对 ErbB-2 和 EGFR 具 有双重抑制活性的 GW5289, GW5945, GW9525 等系列 化合物, 化合物抑制 $\mathrm{EGFR}$ 的 $\mathrm{IC}_{50}$ 值分别为 $0.026,0.022$, $0.029 \mu \mathrm{mol} / \mathrm{L}$, 略低于对照药物 Erlotinib ( $\mathrm{IC}_{50}$ 值为 0.017 $\mu \mathrm{mol} / \mathrm{L}$ )的抑制活性; 抑制 BT474 细胞增殖的 $\mathrm{IC}_{50}$ 值分 别为 $1.16,0.39,0.59 \mu \mathrm{mol} / \mathrm{L}$, 优于对照药物 Erlotinib $\left(\mathrm{IC}_{50}\right.$ 值为 $\left.4.2 \mu \mathrm{mol} / \mathrm{L}\right)$; 抑制 $\mathrm{N} 87$ 细胞增殖的 $\mathrm{IC}_{50}$ 值分别 为 $1.23,0.61,0.80 \mu \mathrm{mol} / \mathrm{L}$, 优于对照药物 Erlotinib $\left(\mathrm{IC}_{50}\right.$ 值为 $5.1 \mu \mathrm{mol} / \mathrm{L}$ ); 抑制 $\mathrm{HN} 5$ 细胞增殖的 $\mathrm{IC}_{50}$ 值分别为 $0.83,0.64,1.13 \mu \mathrm{mol} / \mathrm{L}$, 差于对照药物 Erlotinib $\left(\mathrm{IC}_{50}\right.$ 值 为 $0.18 \mu \mathrm{mol} / \mathrm{L}$ ). 然而, 与正常的人成纤维细胞比较, 3 个化合物对癌细胞增殖抑制的选择性高达 9 47 倍, 说 明该类化合物有开发成广谱癌症治疗药物的可能.<smiles>Cc1nnc(-c2ccc3ncnc(Nc4ccc5c(cnn5Cc5cccc(F)c5)c4)c3c2)o1</smiles><smiles>CS(=O)(=O)CCNCCCCCOc1ccc2ncnc(Nc3ccc4c(cnn4Cc4cccc(F)c4)c3)c2c1</smiles>

GW5945

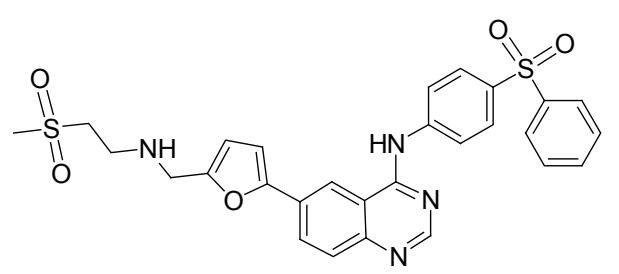

GW9529

2003 年 Gaul 等 ${ }^{[80}$ 报道了一系列 6-噻唑基喹唑啉 33a 33f，该系列化合物抑制 EGFR 的 $\mathrm{IC}_{50}$ 值范围为 $0.008 \sim 0.082 \mu \mathrm{mol} / \mathrm{L}$, 此外, 该系列化合物还可以作为 EGFR 和 ErbB-2 酪氨酸激酶的双重抑制剂, 其 $\mathrm{IC}_{50}$ 值范 围为 $0.011 \sim 0.32 \mu \mathrm{mol} / \mathrm{L}$, 体外抑制 HN-5, N87, BT474 肿瘤细胞增殖的 $\mathrm{IC}_{50}$ 值范围为 $0.089 \sim 7.4 \mu \mathrm{mol} / \mathrm{L}$. 对雌 性 CD-1 小鼠进行活体试验，结果表明化合物对对 HN-5 癌细胞诱发的肿瘤的抑制率为 $43 \% \sim 84 \%$, 对 BT474 癌细胞诱发的肿瘤的抑制率为 33\% $83 \%$.<smiles>[R]Nc1ncnc2ccc(-c3csc(CNCCS(C)(=O)=O)n3)cc12</smiles>

$33 a \sim 33 f$<smiles>[R]=Cc1ccc(F)c(Cl)c1</smiles><smiles>[R]=Cc1ccc(OCc2ccccc2)cc1</smiles><smiles>C=[Ru]Cc1ccc2c(cnn2Cc2ccccc2)c1</smiles><smiles></smiles><smiles>[R]=Cc1ccc(OCc2ccccc2)c(Cl)c1</smiles><smiles>[R]=Nc1ccc(OCc2cccc(F)c2)c(Cl)c1</smiles>

2004 年 Wallace 等 ${ }^{[81]}$ 在专利中报道了 6-位取代基中 含有 $N$-氧基椫的 4-芳胺基喹唑啉化合物. 该系列化合 物抑制 $\mathrm{EGFR}$ 的 $\mathrm{IC}_{50}$ 值均低于 $33 \mathrm{nmol} / \mathrm{L}$, 其中化合物 为 $34 \mathrm{a}$ 对 $\mathrm{EGFR}$ 的 $\mathrm{IC}_{50}$ 值为 $8 \mathrm{nmol} / \mathrm{L}$.

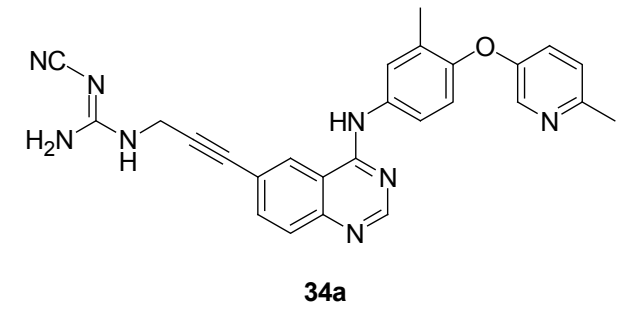

2006 年 Kume 等 ${ }^{[82]}$ 报道了一系列 6-肜醚或肜酯取 代的喹唑啉化合物 $\mathbf{3 5}$, 其中有 49 个化合物均有很好的 抑制 EGFR 的活性, $\mathrm{IC}_{50}$ 值小于 $10 \mathrm{nmol} / \mathrm{L}$, 其中化合物 35a 对 EGFR 的 $\mathrm{IC}_{50}$ 值为 $2.4 \mathrm{nmol} / \mathrm{L}$; 体外活性试验表 明 12 个化合物均有良好的抑制人乳腺导管癌细胞 BT-474 增殖的活性, $\mathrm{IC}_{50}$ 值小于 $10 \mathrm{nmol} / \mathrm{L}$, 其中化合物 35b 对 BT-474 细胞的增殖抑制活性 $\mathrm{IC}_{50}$ 值为 $1.2 \mathrm{nmol} / \mathrm{L}$; 而 35c, 35d 抑制 $\mathrm{EGFR}$ 的 $\mathrm{IC}_{50}$ 值分别为 $43,27 \mathrm{nmol} / \mathrm{L}$, 抑制 BT-474 细胞增殖的 $\mathrm{IC}_{50}$ 值分别为 $26 \mathrm{nmol} / \mathrm{L}, 8$ $\mathrm{nmol} / \mathrm{L}$, 将 35c, 35d 进行雌鼠活体试验, 其在 $20 \mathrm{mg} \cdot$ $\mathrm{kg}^{-1} \cdot \mathrm{d}^{-1}$ 的剂量处理对胃癌的肿瘤体积缩小率分别为 $54 \%, 32 \%$ (治疗组与对照组之比, $\mathrm{T} / \mathrm{C}$ ), $80 \mathrm{mg} \cdot \mathrm{kg}^{-1} \cdot \mathrm{d}^{-1}$ 的剂量下 $\mathrm{T} / \mathrm{C}$ 则分别达到了 $29 \%, 4 \%$. 
<smiles>[R]ON=C(C#CC)c1ccc2ncnc(Nc3ccc(OCc4cccc(F)c4)c(Cl)c3)c2c1</smiles>

$35 a \sim 35 d$

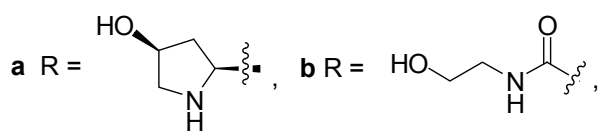<smiles>[R]=C1CCCN1CC(C)(C)CN1CCCC1</smiles>

2007 年 $\mathrm{Liu}$ 等 ${ }^{[83]}$ 合成了一系列含丙炔基的 4-芳胺喹 唑啉化合物 36, 并测试了该系列化合物在体外抑制 EGFR 激酶的活性, 结果表明该类化合物中 $\mathbf{3 6 a}$ 和 $\mathbf{3 6} \mathbf{b}$ 抑制 EGFR 的活性最好, 其 $\mathrm{IC}_{50}$ 值分别为 14.1 和 28.8 $\mathrm{nmol} / \mathrm{L}$ 优于对照药物 Gefitinib $\left(\mathrm{IC}_{50}\right.$ 值 $\left.39.0 \mathrm{nmol} / \mathrm{L}\right)$. 从 活性数据来看, 36a 的活性超过 $\mathbf{3 6 b}$ 的两倍, 这是由于化 合物 36a 在 C-6 位的末端差基与 EGFR 中的 Leu718 上 的氧原子形成了氢键, 提高了该化合物对 EGFR 的亲和 力，从而增强了抑制 EGFR 激酶的活性.<smiles>OCC#Cc1ccc2ncnc(Nc3ccc(F)c(Cl)c3)c2c1</smiles><smiles>C#Cc1cccc(Nc2ncnc3cc(OC)c(OC)cc23)c1</smiles>

$36 \mathrm{~b}$

2010 年 El-Azab 等 ${ }^{[84]}$ 合成了 2-苯基取代的喹唑啉酮 或喹唑啉类化合物 37, 生物活性测试表明化合物抑制 人乳腺癌 $(\mathrm{MCF}-7)$ 细胞增殖的 $\mathrm{IC}_{50}$ 值范围为 $3.35 \sim 6.81$ $\mu \mathrm{g} / \mathrm{mL}$, 其中化合物 37a $~ 37 d$ 表现出广谱的抑制活性, 抑制人肝癌(HEPG2)、人乳腺癌 $(\mathrm{MCF}-7)$ 和人子宫颈癌 (HELA)等细胞增殖的 $\mathrm{IC}_{50}$ 值范围为 $3.35 \sim 5.59 \mu \mathrm{g} / \mathrm{mL}$. 对化合物 $37 \mathrm{c} \sim 37 \mathrm{e}$ 进行分子对接实验, 结果表明化合 物 37c 中的 $\mathrm{CN}$ 基和 37d 中的 $\mathrm{NO}_{2}$ 基与 EGFR 的 Lys-721 形成氢键, 从而增强了化合物的抑制活性, 而化合物 $37 \mathrm{e}$ (抑制这三种癌细胞的 $\mathrm{IC}_{50}$ 值范围为 $9.66 \sim 18.4$ $\mu \mathrm{g} / \mathrm{mL}$ ) 因为没有能够提供与 Lys-721 形成氢键的 $\mathrm{N}$ 骨 架，因此活性远低于化合物 37c 和 37d.

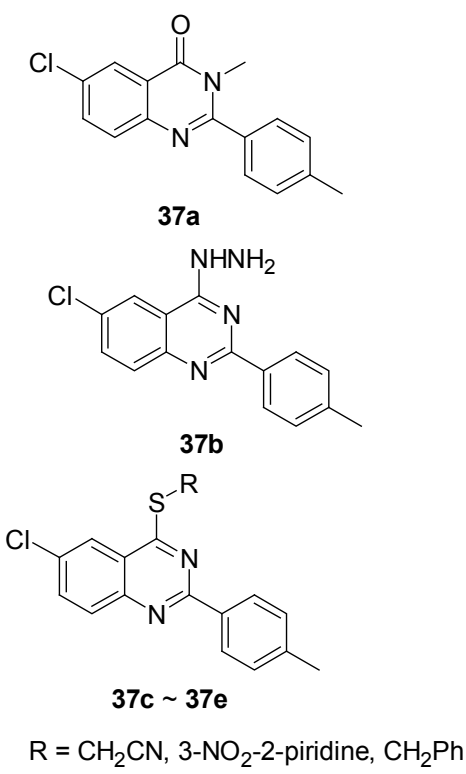

\section{5-烷氧基-4-芳胺喹唑啉}

2005 年 Bradbury 等 ${ }^{[85]}$ 报道了一系列 5-位含四氢吡 咯(哌啶)亚甲氧基的 4-芳胺基喹唑啉化合物 38, 该系列 化合物抑制 $\mathrm{KB}$ 细胞中 $\mathrm{EGFR}$ 的 $\mathrm{IC}_{50}$ 值范围为 $0.001 \sim 5$ $\mu \mathrm{mol} / \mathrm{L}$. 其中代表性化合物 38a $\sim 38 \mathrm{c}$ 抑制 $\mathrm{EGFR}$ 的 $\mathrm{IC}_{50}$ 值分别为 $0.26,33,2.4 \mu \mathrm{mol} / \mathrm{L}$, 具有进一步开发成抗癌 药物的可能.<smiles></smiles>

2006 年 Bradbury ${ }^{[86 ~ 88]}$ 报道了一系列 4-取代吲哚基 胺基喹唑啉化合物 39, 生物活性测试结果表明代表性 
化合物 39a $~ 39 f$ 抑制 $\mathrm{EFGR}$ 的 $\mathrm{IC}_{50}$ 值分别为 $0.78,0.063$, $0.18,0.16,0.31$ 和 $0.061 \mu \mathrm{mol} / \mathrm{L}$, 这些化合物可能适合成 为治疗乳腺癌(MCF-7)的口服药物.<smiles>CC(CN(C)C(=O)CO)Oc1cccc2ncnc(Nc3ccc4c(ccn4Cc4ccccn4)c3)c12</smiles>

$39 a$<smiles>CC(COc1cccc2ncnc(N(C)c3ccc4c(ccn4-c4ccccn4)c3)c12)N(C)C(=O)CO</smiles>

$39 b$<smiles>CN(CCOc1cccc2ncnc(Nc3ccc4c(ccn4Cc4ccccn4)c3)c12)C(=O)CO</smiles>

$39 c$<smiles>CN(CCOc1cccc2ncnc(Nc3ccc4c(ccn4Cc4cscn4)c3)c12)C(=O)CO</smiles>

39d<smiles>O=C(CO)N1CCC[C@H]1COc1cccc2ncnc(Nc3ccc4c(cnn4Cc4ccccn4)c3)c12</smiles>

$39 \mathrm{e}$<smiles>O=C(CO)N1CCCC[C@H]1COc1cccc2ncnc(Nc3ccc4c(cnn4Cc4ccccn4)c3)c12</smiles>

$39 f$

\section{4 其它类型喹唑啉及生物活性}

2004 年 Keenan 和 Shakespear ${ }^{[89]}$ 报道了一类 5-位或 6-位磷取代-4-芳胺基喹唑啉衍生物, 生物活性测试结果 表明代表性化合物 40a 40c 对 EGFR 抑制活性的 $\mathrm{IC}_{50}$ 值范围为 $0.06 \sim 1.6 \mu \mathrm{mol} / \mathrm{L}$, 且对 MDA-468 癌细胞增殖 具有一定的抑制活性, $\mathrm{IC}_{50}$ 值均小于 $50 \mu \mathrm{mol} / \mathrm{L}$.<smiles>[R]Nc1ncnc2cc([R])c([R])cc12</smiles>

40<smiles>COc1cc2ncnc(Nc3ccc(F)c(Cl)c3)c2cc1P(C)(C)=O</smiles><smiles>CCOP(=O)(OCC)c1cc2c(Nc3ccc(F)c(Cl)c3)ncnc2cc1OC</smiles>

$40 b$<smiles>CCOP(C)(=O)c1cc2c(Nc3ccc(F)c(Cl)c3)ncnc2cc1OC(C)(C)C</smiles>

2007 年 Kitano 等 ${ }^{[90]}$ 合成了一系列 4-炔基喹唑啉和 4-烯基喹唑啉化合物 41, 并测试了其抑制 EGFR 激酶的 活性，13 个化合物抑制 $\mathrm{EGFR}$ 的 $\mathrm{IC}_{50}$ 值均小于 30 $\mathrm{nmol} / \mathrm{L}$ ，其中活性最好的 3 个化合物 $41 \mathrm{a} \sim 41 \mathrm{c}$ 的 $\mathrm{IC}_{50}$ 值为 $3,4,5 \mathrm{nmol} / \mathrm{L}$; 化合物 41d 抑制 $\mathrm{EGFR}$ 的 $\mathrm{IC}_{50}$ 值为 $11.7 \mathrm{nmol} / \mathrm{L}$, 将该化合物进行手性拆分后得到的 $(R)-41 \mathrm{~d}\left(\mathrm{IC}_{50}\right.$ 值为 $\left.4.2 \mathrm{nmol} / \mathrm{L}\right)$ 抑制 $\mathrm{EGFR}$ 的活性超出其 对映异构体 $(S)-41 d\left(\mathrm{IC}_{50}\right.$ 值为 $\left.662 \mathrm{nmol} / \mathrm{L}\right)$ 的抑制活性 150 倍.

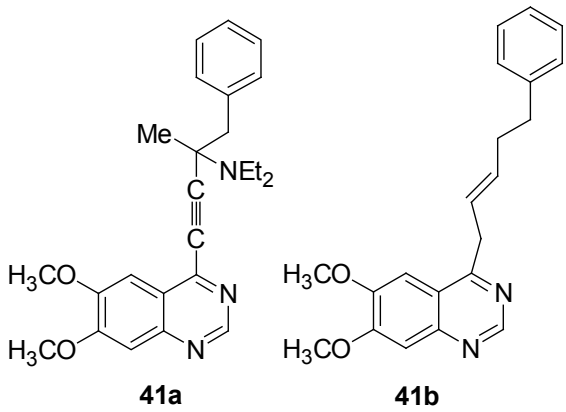



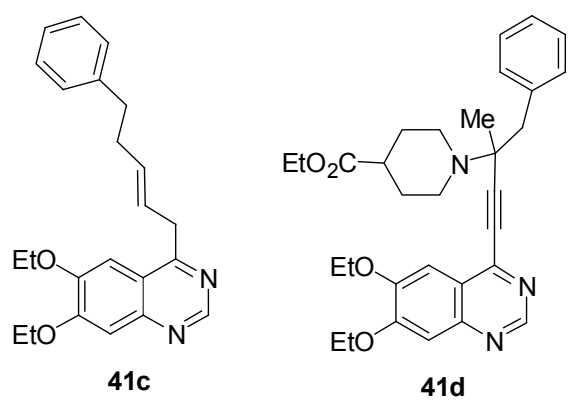

2008 年 Abouzid 和 Shouman ${ }^{[91]}$ 设计合成了一系列 4-磺酰胺基哌嗪(或氨基磺酰基苯胺基)喹唑啉化合物 42, 生物活性测试结果表明该类化合物对人乳腺癌细胞 (MCF-7) 具有良好的体外增殖抑制活性, 其中化合物 42a, 42b, 42c 体外抑制人乳腺癌细胞的 $\mathrm{IC}_{50}$ 值分别为 $1.08,1.98,0.13 \mathrm{nmol} / \mathrm{L}$, 具有极高的抗癌活性.

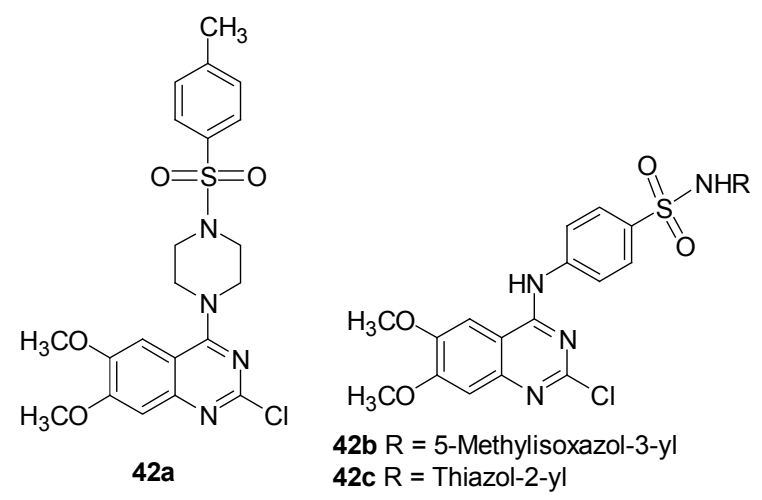

2008 年 San Juan ${ }^{[92]}$ 分析了文献报道中已得到的一 系列化合物 43 的抑制 EGFR 磷酸化的活性 ${ }^{[93,94]}$, 其中活 性最高的化合物 43a 和 43b 抑制 EGFR 的 PIC 50 值均为 8.02 , 具有极高的 EGFR 抑制活性, 分别使用 CoMFA 和 CoMSIA 方法建立了化合物的 3D-QSAR 模型. 在此基 础上, 作者提出, 在吡咯环上引入一个大基团、同时在 喹唑啉环上引入电负性基团、以及在甲酸酯上引入一个 氢键供体都将提高化合物对 EGFR 抑制活性，从而为新 型喹唑啉衍生物的设计提供了新的思路.<smiles>CN(C)CCOCCO</smiles>

43a<smiles>OCCNCc1c[nH]c2cc3ncnc(Nc4cccc(Br)c4)c3cc12</smiles>

43b
2009 年 Zhang 等 ${ }^{[95]}$ 合成了一系列 2,3-二取代-8-芳 胺-3 氢-咪唑并 $[4,5-\mathrm{g}]$ 喹唑啉 44, 对 5 种人类癌细胞(人 肺癌细胞 $\mathrm{A} 549$, 人白血病细胞 K562 和 Molt-4, 人前 列腺癌细胞 PC-3, 人乳腺癌细胞 MDA-MB-231)进行 了体外活性测试, 大部分化合物对这些细胞表现出有效 的抑制活性，对人肺癌细胞 A549 的抑制活性尤其突出, 其中有 11 个化合物活性与对照药物 Gefitinib(抑制 A549 细胞的 $\mathrm{IC}_{50}$ 值为 $13.59 \mu \mathrm{mol} / \mathrm{L}$ )相当甚至优于 Gefitinib, 抑制 $\mathrm{A} 549$ 细胞的 $\mathrm{IC}_{50}$ 值范围为 $2.81 \sim 12.14 \mu \mathrm{mol} / \mathrm{L}$. 采 用流式细胞仪对其中活性最好的 3 个化合物 $\mathbf{4 4 a} \sim \mathbf{4 4 c}$ 进行了细胞周期分析, 通过流动血细胞计数法对 A549 进行细胞周期分析, 发现经 3 个化合物处理的 A549 细 胞有 $80.38 \% \sim 86.28 \%$, 在 $\mathrm{G} 0 / \mathrm{G} 1$ 期内, 意味着化合物能 很大程度使细胞周期停滞在这一阶段, 这一结果与对照 药物 Gefitinib 类似.<smiles>CCCCn1c(C(C)C)nc2cc3c(Nc4ccc(F)cc4)ncnc3cc21</smiles>

\section{5 结论和展望}

本文对近十年来具有抑制 EGFR 活性的喹唑啉类化 合物的生物活性研究进展作了简单的介绍, 从中可以发 现: (1)对具有 EGFR 抑制活性的喹唑啉化合物的修饰主 要集中于喹唑啉环 C-4, C-5, C-6, C-7 位上的取代, 尤其 是 4-芳胺-5,6-二烷氧基喹唑啉和 4-芳胺-6-胺基喹唑啉, 具有良好的抑制 EGFR 的活性. (2)在上述喹唑啉类化合 物中, 喹唑啉环 4-位的取代基团主要是胺类, 尤其是芳 胺和杂环胺类取代活性较高; 但近年来也发展了 C-4 位 为炔基、烯基等取代，也有较好的活性. (3) C-4 位芳胺 的芳环上无取代基或吸电子取代基时，对 EGFR 的抑制 活性普遍远优于供电子取代基，而 4- $N$ 的甲基取代对其 生物活性影响不大. (4)早期的 4-苯胺基喹唑啉主要以可 
逆性抑制剂为主，而近年来，6-位或 7-位含有丙烯酰胺 等可以与 EGFR 的部分氨基酸残基发生 Michael 加成的 不可逆抑制剂正引起越来越多的重视, 不仅活性提高, 且对突变型甚至双突变型 EGFR 都表现出较好的活性. (6)近年来对双重靶标的 4-苯胺基喹唑啉类化合物研究 越来越受到重视. 比如在喹唑啉 6-位上取代三氮脒等, 在保持喹唑啉类化合物对 EGFR 的高效抑制活性的同 时, 还兼具 DNA 损伤等细胞毒功能, 对肿瘤细胞的增 殖会有更好的抑制效果. 此外关于同时作用于 EGFR, erbB2 等靶标的喹唑啉类化合物的报道也日益增多. (7) 当前, 部分喹唑啉类化合物有可能发展成比已上市药物 疗效更好的候选药物品种, 部分化合物已完成一阶段临 床试验. 由于喹唑啉类衍生物有着较多的结构修饰潜 力, 表现出了高效的 EGFR 抑制活性及对肿瘤细胞增殖 的抑制活性，因而具有较强的市场开发潜力. 随着对 EGFR 突变及其抗性机制、喹唑啉类化合物与靶标的相 互作用等研究的不断深入, 相信将会有更安全、高效和 广谱的喹唑啉类医药不断被开发出来, 用于各类肿瘤和 癌症的治疗, 在人类与恶性疾病的斗争中做出重要贡 献.

\section{References}

[1] Aaronson, S. A. Science 1991, 254, 1146.

[2] Ullrich, A.; Schlessinger, J. Cell 1990, 61, 203.

[3] Prenzel, N.; Fischer, O. M.; Streit, S.; Hart, S.; Ullrich, A. Endocr-Relat. Cancer 2001, 8, 11.

[4] Mendelsohn, J.; Baselga, J. J. Clin. Oncol. 2003, 21, 2787.

[5] Minkovsky, N.; Berezov, A. Curr. Opin. Invest. Dr. 2008, 9, 1336.

[6] Hynes, N. E.; Lane, H. A. Nat. Rev. Cancer 2005, 5, 341.

[7] Fry, D. W.; Kraker, A. J.; McMichael, A.; Ambroso, L. A.; Nelson, J. M.; Leopold, W. R.; Connors, R. W.; Bridges, A. J. Science 1994, 265, 1093.

[8] Bridges, A. J. Chem. Rev. 2001, 101, 2541.

[9] Normanno, N.; Maiello, M. R.; De, L. A. J. Cell. Physiol. 2003, 194, 13.

[10] Boschelli, D. H. Curr. Top. Med. Chem. 2002, 2, 1051.

[11] Ciardiello, F. Drugs 2000, 60, 25.

[12] Ciardiello, F.; Caputo, R.; Bianco, R.; Damiano, V.; Pomatico, G.; Placido, S. D.; Bianco, A. R.; Tortora, G. Clin. Cancer Res. 2000, 6, 2053.

[13] Moasser, M. M.; Basso, A.; Averbuch, S. D.; Rosen, N. Cancer Res. 2001, 61, 7184.

[14] Friedmann, B.; Caplin, M.; Hartley, J. A.; Hochhauser, D. Clin. Cancer Res. 2004, 10, 6476.

[15] Chang, G.-C.; Hsu, S.-L.; Tsai, J.-R.; Liang, F.-P.; Lin, S.-Y.; Sheu, G.-T.; Chen, C.-Y. Biochem. Pharmacol. 2004, 68, 1453.

[16] Lee, E. J.; Whang, J. H.; Jeon, N. K.; Kim, J. Ann. N. Y. Acad. Sci. 2007, 1095, 113.

[17] Wakeling, A. E. Curr. Opin. Pharmacol. 2002, 2, 382.

[18] Ashkenazi, A. J. WO 2006089015, 2006 [Chem. Abstr. 2006, 145, 263270].

[19] Mark, B.; Hans, S. WO 2007047489, 2007 [Chem. Abstr. 2007, 146, 468446]

[20] Rosetti, A.; Tesei, A.; Ulivi, P.; Fabbri, F.; Vannini, I.; Brigliadori,
G.; Granato, A. M.; Amadori, D.; Zoli, W. Cancer Biol. Ther. 2005 , 4, 1089.

[21] Sabitha, K.; Kishore, K. M.; Kaiser, J. J. Mol. Graphics Modell. 2008, 27, 244.

[22] Huq, F. Int. J. Pure Appl. Chem. 2007, 2, 281

[23] Kobayashi, S.; Boggon, T. J.; Dayaram, T.; Janne, P. A.; Kocher, O.; Meyerson, M.; Johnson, B. E.; Eck, M. J.; Tenen, D. G.; Halmos, B. N. Engl. J. Med. 2005, 352, 786.

[24] Sequist, L. V.; Bell, D. W.; Lynch, T. J.; Haber, D. A. J. Clin. Oncol. 2007, 25, 587.

[25] Flavio, S. WO 2008034776, 2008 [Chem. Abstr. 2008, 148, 394375].

[26] Vicentini, C.; Festuccia, C.; Gravina, G. L.; Angelucci, A.; Marronaro, A.; Bologna, M. J. Cancer Res. Clin. 2003, 129, 165.

[27] Shreder, K. R.; Wong, M. S.; Nomanbhoy, T.; Leventhal, P. S.; Fuller, S. R. Org. Lett. 2004, 6, 3715.

[28] Hennequin, L. F. A.; Halsall, C. T. WO 2005026150, 2005 [Chem. Abstr. 2005, 142, 336378].

[29] Bradbury, R. H.; Hennequin, L. F. A.; Barlaam, B. C. $W O$ 2005028469, 2005 [Chem. Abstr. 2005, 142, 355277].

[30] Barlaam, B. C.; Halsall, C. T.; Hennequin, L. F. A. $W O$ 2005030765, 2005 [Chem. Abstr. 2005, 142, 355279].

[31] Kiguchi, K.; Ruffino, L.; Kawamoto, T.; Ajiki, T.; DiGiovanni, Clin. Cancer. Res. 2005, 11, 5572.

[32] Hennequin, L. F. A.; Halsall, C. T. WO 2005026157, 2005 [Chem Abstr. 2005, 142, 336384].

[33] Halsall, C. T.; Hennequin, L. F. A.; Plowright, A. T.; Storey, R.; Lennon, K. WO 2006090163, 2006 [Chem. Abstr. 2006, 145, 293085].

[34] Xu, C.-L.; Guo, Z.-X.; Zhang, Y.-D.; Zhao, F.-K.; Xu, G.-J.; Yu, Y.-P. Chin. Pharm. J. 2007, 42, 1748 (in Chinese). (许传莲, 郭宗香, 章砚东, 赵辅昆, 许根俊, 俞永平, 中国药学 杂志 2007, 42, 1748.)

[35] Breza, N.; Pato, J.; Orfi, L.; Hegymegi-Barakonyi, B.; Banhegyi, P.; Varkondi, E.; Borbely, G.; Petak, I.; Keri, G. J. Recept. Signal Transduction Res. 2008, 28, 361.

[36] Lee, K.-O.; Cha, M.-Y.; Kim, M.-R.; Jung, Y.-H.; Lee, C.-G.; Kim, S.-Y.; Bang, K.; Park, B.-W.; Choi, B.-I.; Chae, Y.-J.; Ko, M.-Y.; Kim, H.-K.; Ahn, Y.-G.; Kim, M.-S.; Lee, G.-S. WO 2008150118 , 2008 [Chem. Abstr. 2009, 150, 35390].

[37] Schettino, C.; Bareschino, M. A.; Ricci, V.; Ciardiello, F. Expert Rev. Resp. Med. 2008, 2(2), 167

[38] Hidalgo, M.; Siu, L. L.; Nemunaitis, J.; Rizzo, J.; Hammond, L. A.; Takimoto, C. Eckhardit, S. G.; Tolcher, A.; Britten, C. D.; Denis, L.; Ferrante, K.; Von Hoff, D. D.; Silberman, S.; Rowinsky, E. K. J. Clin. Oncol. 2001, 19, 3267.

[39] Siu, L. L.; Hidalgo, M.; Nemunaitis, J.; Rizzo, J.; Moczygemba, J.; Eckhardt, S. G.; Tolcher, A.; Smith, L.; Hammond, L.; Blackburn, A.; Tensfeldt, T.; Silberman, S.; Von Hoff, D. D. Proc. Am. Soc. Clin. Oncol. 1999, 18, 1498.

[40] Jimenez, U.; Gurpide, A.; Isla, D.; Garcia-Campelo, R.; Firvida, J.; Vinolas, N.; Jaen, A.; Montalar, J.; Regueiro, P.; Massuti, B. Proc. Am. Soc. Clin. Oncol. 2007, 26, 7639.

[41] Groen, H. J.; Smit, E. F.; Dingemans, A. Proc. Am. Soc. Clin. Oncol. 2007, 26, 7625 .

[42] Smith, J. Clin. Ther. 2005, 27, 1513.

[43] Ban, H. S.; Onagi, S.; Uno, M.; Nabeyama, W.; Nakamura, H. Chem. Med. Chem. 2008, 3, 1094.

[44] Lueth, A.; Loewe, W. J. Heterocycl. Chem. 2008, 45, 703.

[45] Qian, C.-G.; Cai, X. WO 2008033748, 2008 [Chem. Abstr. 2008, 148, 355812].

[46] Qian, C.-G.; Cai, X.; Gould, S.; Zhai, H.-X. WO 2008033749, 2008 
[Chem. Abstr. 2008, 148, 355813].

[47] Cai, X.; Qian, C.-G.; Zhai, H.-X.; Bao, R. US 20090111772, 2009 [Chem. Abstr. 2009, 116, 524181].

[48] Cai, X.; Qian, C.-G.; Zhai, H.-X. WO 2009035718, 2009 [Chem. Abstr. 2009, 150, 352185].

[49] Chandregowda, V.; Kush, A. K.; Reddy, G. C. Eur. J. Med. Chem. 2009, 44, 3046.

[50] Hatti, K. S.; Chandregowda, V.; Rao, G. V.; Kush, A.; Reddy, G. C. J. Proteomics Bioinform. 2009, 2, 126.

[51] Ban, H. S.; Tanaka, Y. W.; Hatori, N. M.; Nakamura, H. Bioorg. Med. Chem. 2010, 18, 870.

[52] Chilin, A.; Conconi, M. T.; Marzaro, G.; Guiotto, A.; Urbani, L.; Tonus, F.; Parnigotto, P. J. Med. Chem. 2010, 53, 1862.

[53] Garofalo, A.; Goossens, L.; Lemoine, A.; Ravez, S.; Six, P.; Howsam, M.; Farce, A.; Depreux, P. Med. Chem. Commun. 2011, $2,65$.

[54] Ahn, Y.-G.; Kim, J.-W.; Bang, K.-C.; Park, B.-W.; Kim, S.-Y.; Lee, K.; Lee, K.; Ko, M.-S.; Kim, H.-K.; Kim, Y.-H.; Kim, M.-S.; Lee, G.-S. WO 2007055514, 2007 [Chem. Abstr. 2007, 146, 501078].

[55] Dissoki, S.; Aviv, Y.; Laky, D.; Abourbeh, G.; Levitzki, A.; Mishani, E. Appl. Radiat. Isot. 2007, 65, 1140.

[56] Wissner, A.; Fraser, H. L.; Ingalls, C. L.; Dushin, R. G.; Floyd, M. B.; Cheung, K.; Nittoli, T.; Ravi, M. R.; Tan, X.; Loganzo, F. Bioorg. Med. Chem. 2007, 15, 3635.

[57] Li, D.; Ambrogio, L.; Shimamura, T.; Kubo, S.; Takahashi, M.; Chirieac, L. R.; Padera, R. F.; Shapiro, G. I.; Baum, A.; Himmelsbach, F.; Rettig, W. J.; Meyerson, M.; Solca, F.; Greulich, H.; Wong, K.-K. Oncogene 2008, 27, 4702.

[58] Frank, H.; Elik, L.; Stefan, B.; Birgit, J.; Elike, B.; Flavio, S. WO 02/50043, 2002 [Chem. Abstr. 2002, 137, 63250].

[59] Eskens, F. A.; Mom, C. H.; Planting, A. S.; Gietema, J. A.; Amelsberg, A.; Huisman, H.; Van Doorn, L.; Burqer, H.; Stopfer, P.; Verweij, J.; De Vries, E. G. Br. J. Cancer. 2008, $98,80$.

[60] Hennequin, L. F. A.; Halsall, C. T. WO 2005026156, 2005 [Chem. Abstr. 2005, 142, 336383].

[61] Delouvrie, B.; Harris, C. S.; Hennequin, L. F. A.; Halsall, C. T.; Pease, J. E.; Smith, P. M. WO 2005075439, 2005 [Chem. Abstr. 2005, 143, 230185].

[62] Tsou, H. R.; Mamuya, N.; Johnson, B. D.; Reich, M. F.; Gruber, B. C.; Ye, F.; Nilakantan, R.; Shen, R.; Discafani, C.; DeBlanc, R.; Davis, R.; Koehn, F. E.; Greenberger, L. M.; Wang, Y.-F.; Wissner, A. J. Med. Chem. 2001, 44, 2719.

[63] Qiu, Q.; Dudouit, F.; Banerjee, R.; McNamee, J. P.; Jean-Claude, B. J. Prostate 2004, 59, 13.

[64] Mishani, E.; Abourbeh, G.; Rozen, Y.; Jacobson, O.; Laky, D.; Ben D. I.; Levitzki, A.; Shaul, M. Nucl. Med. Biol. 2004, 31, 469.

[65] Ben-David, I.; Rozen, Y.; Ortu, G.; Mishani, E. Appl. Radiat. Isot. 2003, 58, 209.

[66] Matheson, S. L.; McNamee, J. P.; Wang, T.; Alaoui-Jamali, M. A.; Tari, A. M.; Jean-Claude, B. J. J. Pharmacol. Exp. Ther. 2004, 311, 1163.

[67] Brahimi, F.; Rachid, Z.; McNamee, J. P.; Alaoui-Jamali, M. A.; Tari, A. M.; Jean-Claude, B. J. Biochem. Pharmacol. 2005, 70, 511.

[68] Domarkas, J.; Dudouit, F.; Williams, C.; Qiyu, Q.; Banerjee, R.; Brahimi, F.; Jean-Claude, B. J. J. Med. Chem. 2006, 49, 3544.

[69] Ham, Y. J.; Gong, J. H.; Cha, M. Y.; Kim, J. W.; Kim, M. S.; Kim, E. Y.; Song, J. Y.; Kim, C. I.; Kim, S. Y.; Lee, G. S. WO 2006071017, 2006 [Chem. Abstr. 2006, 145, 124597].

[70] Fernandes, C.; Oliveira, C.; Gano, L.; Bourkoula, A.; Pirmettis, I.; Santos, I. Bioorg. Med. Chem. 2007, 15, 3974.

[71] Blair, J. A.; Rauh, D.; Kung, C.; Yun, Cai-H.; Fan, Qi-W.; Rode,
H.; Zhang, C.; Eck, M. J.; Weiss, W. A.; Shokat, K. M. Nat. Chem. Biol. 2007, 3, 229.

[72] Michalczyk, A.; Klueter, S.; Rode, H. B.; Simard, J. R.; Gruetter, C.; Rabiller, M.; Rauh, D. Bioorg. Med. Chem. 2008, 16, 3482.

[73] Bourkoula, A.; Paravatou-Petsotas, M.; Papadopoulos, A.; Santos, I.; Pietzsch, H. J.; Livaniou, E.; Pelecanou, M.; Papadopoulos, M.; Pirmettis, I. Eur. J. Med. Chem. 2009, 44, 4021.

[74] Rachid, Z.; MacPhee, M.; Williams, C.; Todorova, M.; Jean-Claude, B. J. Bioorg. Med. Chem. Lett. 2009, 19, 5505.

[75] Banerjee, R.; Rachid, Z.; McNamee, J.; Jean-Claude, B. J. J. Med. Chem. 2003, 46, 5546.

[76] Rachid, Z.; Brahimi, F.; Katsoulas, A.; Teoh, N.; Jean-Claude, B. J. J. Med. Chem. 2003, 46, 4313.

[77] Bradbury, R. H.; Hennequin, L. F. A.; Kettle, J. G.; Barlaam, B. C. WO 2005026151, 2005 [Chem. Abstr. 2005, 142, 336379].

[78] Ban, H. S.; Usui, T.; Nabeyama, W.; Morita, H.; Fukuzawa, K.; Nakamura, H. Org. Biomol. Chem. 2009, 7, 4415.

[79] Rusnak, D. W.; Affleck, K.; Cockerill, S. G.; Stubberfield, C.; Harris, R.; Page, M.; Smith, K. J.; Guntrip, S. B.; Carter, M. C.; Shaw, R. J.; Jowett, A.; Stables, J.; Topley, P.; Wood, E. R.; Brignola, P. S.; Kadwell, S. H.; Reep, B. R.; Mullin, R. J.; Alligood, K. J.; Keith, B. R.; Crosby, R. M.; Murray, D. M.; Knight, W. B.; Gilmer, T. M.; Lackey, K. Cancer. Res. 2001, 61, 7196.

[80] Gaul, M. D.; Guo, Y.; Affleck, K.; Cockerill, G. S.; Gilmer, T. M.; Griffin, R. J.; Guntrip, S.; Keith, B. R.; Knight, W. B.; Mullin, R. J.; Murray, D. M.; Rusnak, D. W.; Smith, K.; Tadepalli, S.; Wood, E. R.; Lackey, K. Bioorg. Med. Chem. Lett. 2003, 13, 637.

[81] Wallace, E.; Topalov, G.; Zhao, Q. WO 2004046101, 2004 [Chem. Abstr. 2004, 141, 7132].

[82] Kume, M.; Matsuo, K.; Omori, N.; Takayama, M.; Omori, A.; Endo, T. WO 2006090717, 2006 [Chem. Abstr. 2006, 145, 515901]

[83] Liu, L.-T.; Yuan, T.-T.; Liu, H.-H.; Chen, S.-F.; Wu, Y.-T. Bioorg. Med. Chem. Lett. 2007, 17, 6373.

[84] El-Azab, A. S.; Al-Omar, M. A.; Abdel-Aziz, A. A.-M.; Abdel-Aziz, N. I.; El-Sayed, M. A.-A.; Aleisa, A. M.; Sayed-Ahmed, M. M.; Abdel-Hamide, S. G. Eur. J. Med. Chem. 2010, 45, 4188.

[85] Bradbury, R. H.; Hennequin, L. F. A.; Kettle, J. G. WO 005026152 , 2005 [Chem. Abstr. 2005, 142, 336380].

[86] Bradbury, R. H. WO 2006117521, 2006 [Chem. Abstr. 2006, 145, 489260].

[87] Bradbury, R. H. WO 2006117523, 2006 [Chem. Abstr. 2006, 145, 489259].

[88] Bradbury, R. H. WO 2006092573, 2006 [Chem. Abstr. 2006, 145, 315007].

[89] Keenan, T. P.; Shakespear, W. C. WO 2004058267, 2004 [Chem. Abstr. 2004, 141, 123644].

[90] Kitano, Y.; Suzuki, T.; Kawahara, E.; Yamazaki, T. Bioorg. Med. Chem. Lett. 2007, 17, 5863.

[91] Abouzid, K.; Shouman, S. Bioorg. Med. Chem. 2008, 16, 7543.

[92] San Juan, A. A. Eur. J. Med. Chem. 2008, 43, 781.

[93] Palmer, B. D.; Trumpp-Kallmeyer, S.; Fry, D. W.; Nelson, J. M.; Showalter, H.; Denny, W. A. J. Med. Chem. 1997, 40, 1519.

[94] Wissner, A.; Berger, D. M.; Boschelli, D. H.; Floyd, M. B.; Greenberger, L. M.; Gruber, B. C.; Johnson, B. D.; Mamuya, N.; Nilakantan, R.; Reich, M. F.; Shen, R.; Tsou, H.-R.; Upeslacis, E.; Wang, Y.-F.; Wu, B.; Ye, F.; Z hang, N. J. Med. Chem. 2000, 43, 3244.

[95] Zhang, Y.-D.; Chen, Z.; Lou, Y.-J.; Yu, Y.-P. Eur. J. Med. Chem. 2009, 44, 448.

(Qin, X.) 